Review

\title{
Drug-Drug Interactions in Elderly Patients with Potentially Inappropriate Medications in Primary Care, Nursing Home and Hospital Settings: A Systematic Review and a Preliminary Study
}

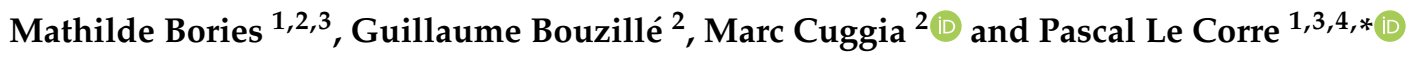 \\ 1 Pôle Pharmacie, Service Hospitalo-Universitaire de Pharmacie, CHU de Rennes, 35033 Rennes, France; \\ mathilde.bories@chu-rennes.fr \\ 2 Univ Rennes, CHU Rennes, INSERM, LTSI-UMR 1099, F-35000 Rennes, France; \\ guillaume.bouzille@univ-rennes1.fr (G.B.); marc.cuggia@univ-rennes1.fr (M.C.) \\ 3 Laboratoire de Biopharmacie et Pharmacie Clinique, Faculté de Pharmacie, Université de Rennes 1, \\ 35043 Rennes, France \\ 4 Univ Rennes, CHU Rennes, Inserm, EHESP, Irset (Institut de recherche en santé, environnement et \\ travail)-UMR_S 1085, F-35000 Rennes, France \\ * Correspondence: pascal.le-corre@univ-rennes1.fr
}

\section{check for}

updates

Citation: Bories, M.; Bouzillé, G.; Cuggia, M.; Le Corre, P. Drug-Drug Interactions in Elderly Patients with Potentially Inappropriate Medications in Primary Care, Nursing Home and Hospital Settings: A Systematic Review and a Preliminary Study. Pharmaceutics 2021, 13, 266. https://doi.org/ $10.3390 /$ pharmaceutics13020266

Academic Editor: Neal M. Davies

Received: 14 January 2021

Accepted: 11 February 2021

Published: 16 February 2021

Publisher's Note: MDPI stays neutral with regard to jurisdictional claims in published maps and institutional affiliations.

Copyright: (C) 2021 by the authors. Licensee MDPI, Basel, Switzerland. This article is an open access article distributed under the terms and conditions of the Creative Commons Attribution (CC BY) license (https:/ / creativecommons.org/licenses/by/ $4.0 /)$.
Abstract: Drug-drug interactions (DDI) occurring with potentially inappropriate medications (PIM) are additional risk factors that may increase the inappropriate character of PIM. The aim of this study was (1) to describe the prevalence and severity of DDI in patients with PIM and (2) to evaluate the DDI specifically regarding PIM. This systematic review is based on a search carried out on PubMed and Web-of-Science from inception to June 30, 2020. We extracted data of original studies that assessed the prevalence of both DDI and PIM in elderly patients in primary care, nursing home and hospital settings. Four hundred and forty unique studies were identified: 91 were included in the qualitative analysis and 66 were included in the quantitative analysis. The prevalence of PIM in primary care, nursing home and hospital were $19.1 \%$ (95\% confidence intervals (CI): $15.1-23.0 \%$ ), 29.7\% (95\% CI: 27.8-31.6\%) and 44.6\% (95\% CI: 28.3-60.9\%), respectively. Clinically significant severe risk-rated DDI averaged 28.9\% (95\% CI: 17.2-40.6), in a hospital setting; and were approximately 7-to-9 lower in primary care and nursing home, respectively. Surprisingly, only four of these studies investigated DDI involving specifically PIM. Hence, given the high prevalence of severe DDI in patients with PIM, further investigations should be carried out on DDI involving specifically PIM which may increase their inappropriate character, and the risk of adverse drug reactions.

Keywords: drug interactions; aged; potentially inappropriate medications; hospital; nursing home; primary care

\section{Introduction}

A drug-drug interaction (DDI) is usually defined as a clinically significant unintended modification in the exposure and/or response to a medication (i.e., victim) that occurs with the co-administration of another medication (i.e., perpetrator) [1]. Studies in the field of DDIs usually refer to a potential DDI, defined as the co-prescription of two medications known to interact, that may occur in exposed patients. DDIs can be categorized as either of pharmacokinetic origin (i.e., modification in exposure) or of pharmacodynamic origin (i.e., modification in response) [2]. A pharmacodynamic DDI results from the concomitant administration of medications that have the same sites of action, leading to additive, synergistic or antagonistic effects altering the drug effect with usually no apparent alteration in drug exposure. A pharmacokinetic DDI results from alterations in the processes involved in drug disposition when two drugs are co-administered. Basically, these DDI are due to either metabolic enzymes and/or transporters localized in membranes and tissues involved 
in absorption, distribution, metabolism or drug excretion, leading to apparent alteration in drug exposure.

Metabolism mediated by cytochrome P450 isoenzymes (principally CYP450 1A2, 2C9, 2C19, 2D6 and 3A4) is known to play a major part in the biotransformation of drugs in vivo, and in the interindividual variability in drug response. Drug transporters are categorized into two superfamilies: ATP binding cassette (ABC) transporters and solute carrier transporters (SLC) [3]. Among transporters, P-gp is the efflux transporter most commonly involved in DDI [4] The clinical consequences of drug interactions depend on the magnitude of their impact on the systemic exposure (i.e., area under the curve (AUC)). The intensity (strong, moderate or weak) of metabolic inhibition or induction is defined by the Food and Drug Administration (FDA) based on the impact on AUC [5]. Indeed, a strong inhibitor for a specific CYP is defined as an inhibitor that increases the AUC of a substrate for a more than 5 -fold, or more than $80 \%$, decrease in clearance [5]. For moderate and weak inhibitors, the increase is 2-fold to 5-fold, and 1.25-fold to 2-fold, respectively. An inhibition is a quite immediate phenomenon (occurring in the $24 \mathrm{~h}$ post dosing) while an induction requires the synthesis of new proteins (enzymes or transporters), reaching its maximum at around 7 to 10 days [5]. Thus, in clinical practice, we have to pay particular attention to inhibition mechanisms at either enzyme level or transporter level.

The risk of DDI is higher in elderly patients since they have many co-morbidities that exposed them to polymedications. The risk of DDI is potentially increased by age-related modifications in drug pharmacokinetics and pharmacodynamics [6]. Prevention of DDI in the elderly should be integrated in a medication action plan that not only assesses DDI but also drug-disease interactions, identification and reconsideration of high risk therapy and adjustment for organ elimination [7]. Care pathways of elderly people involve several settings from primary care to nursing home and hospital, with different complexity in the therapeutic management depending on the degree of severity of the diseases.

In a systematic review, DDI was identified as a significant cause of ADR-related hospitalizations with a median DDI prevalence rate of $22.2 \%$ and $8.9 \%$ for hospital admission and hospital visits, respectively [8]. In a prospective study (Effets indésirables des Médicaments: Incidence et Risque (EMIR) study, $n=2692$ admissions), the incidence of hospital admissions related to ADRs was 3.6\% [9] with 29.9\% of these ADR-related hospitalizations resulting from DDI.

Within elderly people exposed to polymedications, prescription of potentially inappropriate medications (PIMs) is an issue. PIMs correspond to medications with different characteristics: i.e., medications that are potentially inappropriate in most older adults, medications that are potentially inappropriate in older adults with certain conditions, medications that should be used with caution, potentially clinically important DDI to be avoided in older adults and medications that should be avoided or have their dosage reduced based on kidney function [10]. With regard to DDI, it should be kept in mind that Beers classification only considers a limited number of potentially clinically significant interactions. Hence, the prevalence of clinically significant interactions in elderly adults is obviously higher than estimated by Beers criteria [10]. Within all the DDI occurring in the elderly in general, DDI occurring specifically with PIM as victim drug is an issue since these DDI could enhance their inappropriate character. Indeed, the inhibition in drug metabolism or transporter-dependent elimination increases the systemic exposure of a PIM and may lead to increase in incidence and/or severity of ADRs and their consequences (i.e., re-hospitalization and/or mortality). Hence, the knowledge of the prevalence of DDI occurring specifically with PIM as victim drugs in elderly patients should be considered as a public health subject, and as of interest for the health professionals in drawing their attention to these specific DDI.

The aim of this study was to perform a systematic review in hospital, nursing home and primary care settings to evaluate the prevalence and severity of DDI occurring in elderly patients for whom PIM are prescribed, and then to evaluate the DDI involving specifically PIM. 


\section{Materials and Methods}

This review was performed according to the Preferred Reporting Items for Systematic Reviews and meta-analyses (PRISMA) statement. The PRISMA check list [11] and PRISMA flow chart are available in Supplementary material 1 and 2.

\subsection{Search Strategy}

We conducted a systematic search of the published peer-reviewed literature in Pubmed and Web of Science databases. Duplicate manuscripts were removed after exporting references in Zotero reference management software (www.zotero.org (accessed on 13 February 2021)).

A literature search was conducted using Medical Subject Headings (MeSH) terms and text words around the topic of drug-drug interactions and potentially inappropriate medication use in older people.

The literature search was carried out from inception to June 1, 2020 without language restrictions, and non-English publications were translated prior to data extraction. Details of the full search strategy are available in Supplementary material 3.

\subsection{Eligibility Criteria}

Original studies reporting the prevalence of DDI and potentially inappropriate medications.

Studies were considered eligible to be included in this review if they provided the age of involved patients. Looking at patient's characteristics, most of the studies focused on patients older than 65 years old. However, two studies selected patients above 60 years old (Brazil and Pakistan). Our choice to include them in this review is based on the WHO definition of an older person in developing countries which has set the threshold at 60 years old for these countries [12]. The following exclusion criteria were applied: duplicate studies, letters, reviews.

\subsection{Study Selection}

Two authors (MB and PLC) independently assessed the publications for inclusion in the review. The titles and abstracts were screened to identify the potentially relevant studies aiming at determining the prevalence of DDI and of PIM in older patients. The full-text copies for the studies that apparently met the criteria as well as those for which an uncertainty existed were retrieved for an independent review by the two authors (MB and PLC). If the two authors did not reach agreement through discussion about a paper to be included, we consulted a third study author (MC) to resolve any disagreement.

\subsection{Data Extraction and Synthesis}

Two review authors (MB, PLC) independently extracted relevant data from full-text articles using a predetermined data extraction form. In case of disagreement, the resolution was obtained by discussion between the two review authors, and a third author (MC) was consulted if necessary.

The following items were included in the extraction form: first author, year, country (ies) of origin, age of patients (mean, threshold if mean non available), study setting (primary care or medical institution), sample size, typology of patients (general population or specific disease), study style, source of data, software or tool used for checking prevalence of PIM and DDI, mean number of medications.

Data were collected from the publications directly as reported or indirectly (i.e., after calculation from the raw data). Any missing data were requested from the study authors.

\subsection{Data Analyses}

The prevalence of DDI and of PIM corresponds to the percentage of patients that had at least one DDI or one PIM.

We decided to report in detail only the DDI considered as clinically significant; that is, DDI with major and moderate risk. There was a diversity of tools used to check the DDI, and the risk rating classification of DDI differed between some of them. Hence, we 
have carried out a mapping of the different DDI checker tools to integrate only DDI we considered as major and moderate risk according to the description that was reported by each tool.

We made the following comparisons:

(a) Prevalence of each parameter (PIM and DDI with severe and moderate risks) reported by the most used tools (using reported mean) without consideration of settings, in a first time. Then, we made the same comparison using computed weighted mean and $95 \%$ CI for the most used tools to evaluate potential differences between settings.

(b) Prevalence of PIM and DDI (with severe and moderate risks) in each setting (using computed weighted mean).

(c) Polymedication between settings (number of prescribed medications per patient reported as the mean).

\subsection{Statistics}

As a result of differences in the number of patients involved the studies we computed a weighted mean for each parameter taking into account the sample size. We used the soymean and confint functions from the R survey package to compute weighted means and $95 \%$ confidence intervals (CI95\%) [13]. Statistical analyses were performed on data from articles focusing on the general population using Shapiro Wilk and Kruskal Wallis tests. For pairwise comparisons of the prevalence of PIM and DDI (with severe and moderate risks) in each setting, a false discovery rate correction for alpha risk was used. Statistical analysis was performed using R software (version 3.6.3). A $p$-value $<0.05$ was considered significant.

\subsection{Mapping of DDI Risk Classification}

Since there are variations in the classification of DDI among DDI softwares, we made a mapping of the DDI risk classification based on the descriptions given by the providers. We retained and classified the DDI we considered major (e.g., contra-indicated/serious/life threatening) or moderate (e.g., use with caution).

\subsection{Data Extraction from Hospital Clinical Data Center}

In the course of this review, we noticed that very few studies were dealing with DDI involving specifically PIM so that we decided to perform a preliminary study within our university hospital clinical data warehouse (CDW) [14]. In order to estimate the prevalence of DDI specifically with PIM, we have chosen 6 PIM from different therapeutic areas (tramadol, apixaban, digoxine, clozapine, glimepiride and quetiapine) with a narrow therapeutic index that are metabolized and/or transported by the most common pathways involved in drug pharmacokinetics (CYP450 isoenzymes: 1A2, 2C9, 2C19, 2D6, 3A4-Transporter: P-gp). For each PIM (as substrate), we have selected some interacting medications known to lead to a DDI rated as moderate or major intensity. This led to a panel of 15 DDI whose prevalence were screened in electronic health records of elderly patients from our CDW (hospitalized in 2019).

\section{Results}

\subsection{Study Selection}

The literature search identified 212 studies in Pubmed and 298 in Web of Science databases leading to 440 studies after removal of duplicate studies. After titles and abstract screening, full-text articles were assessed for legibility. The flow chart is depicted in Supplementary Material 2.

\subsection{Study Characteristics}

Three study groups were created according to the study setting: nursing home $(n=20)$, primary care $(n=38)$ or hospital settings $(n=33)$. Among these 91 studies, 66 focused on 
general population and 25 on specific populations (population with specific medications or specific diseases, e.g., oncology, psychiatric disease, etc.).

Included studies were published from 1994 to 2019. Most of the studies from general population were conducted in Europe $(n=38)$ and North America $(n=13)$. However, other countries from Asia $(n=8)$ or South America $(n=7)$ have also published results. Further details on the characteristics of the studies and the tools used are available as Supplementary Material 4.

Tables 1-3 describe the characteristics of each study evaluated in this review for nursing home, primary care and hospital settings, respectively.

\subsection{Mapping of DDI Checker}

Our study showed that different DDI checker tools are used in different ways in risk rating DDIs (Tables 1-3). The mapping of severity classifications of the different tools that were used is presented in Table 4. Among DDI checker tools, Micromedex and Lexicomp were most frequently used ( $n=17,18 \%$ and $n=10,10 \%$. respectively). The Swedish Physicians' Desk Reference tool was also frequently used, especially as a result of numerous studies $(n=9)$ performed in Sweden in primary care and nursing home settings. Based on these elements, comparison between studies is not straightforward as a result of difference in DDI detection.

\subsection{Potentially Inappropriate Medications}

We checked a potential difference between the tools used to identify PIMs by comparing Beers Criteria, STOPP/START, Swedish National Board of Health and Welfare, Fick list and NORGEP that were the most used tools $(47 \%, 11 \%, 11 \%, 8 \%$ and $5 \%$, respectively). The average prevalence reported by using these tools were $38.6 \%, 60.4 \%, 25.0 \%, 29.2 \%$ and $26 \%$, respectively. Without consideration of settings, the distribution was not normal $(p=0.0018)$. We found a significant difference between the tools $(p=0.020)$ in their ability to detect PIMs.

Since Beers Criteria was the most used tool in the general population ( $n=35,47 \%)$, we made a comparison of the prevalence of PIM according to Beers between the settings. The prevalence of PIMs in primary care, nursing home and hospital were $26.1 \%$ (95\% CI: 25.8-26.4), 20.1\% (95\% CI: 1.6-38.5) and 43.9\% (95\% CI: 23.2-64.5), respectively. Differences between the settings were found $(p=0.0062)$.

Without tool consideration, the weighted mean prevalence of PIM in primary care, nursing home and hospital was: 19.1\% (95\% CI: 15.1-23.0\%), 29.7\% (95\% CI: $27.8-31.6 \%$ ) and $44.6 \%$ (95\% CI: $28.3-60.9 \%)$. The Kruskal-Wallis test used to compare the prevalence of PIM between settings showed significant differences $(p=0.0027)$. Using pairwise comparisons, significant differences were found between nursing homes and primary care $(p=0.0029)$ and between hospital and primary care $(p=0.0037)$ but not between nursing home and hospital $(p=0.4604)$.

The prevalence of PIM in specific populations of patients and in the different settings is presented in Tables 1-3. In the specific populations, given the diversity of diseases encountered (especially mental health diseases in nursing home and oncology in hospital settings), comparison between settings is not relevant. However, it should be noticed that PIM prevalence in the specific populations was higher than in the general populations except for hospital setting (35.0\% in specific population vs. $44.6 \%$ in the general population).

\subsection{Polymedication}

Polymedication did not display a normal distribution $(p=0.023)$ and revealed no differences between settings $(p=0.6741)$. Indeed, the number of medications per patient in primary care, nursing home and hospital was $7.07,7.02$ and 7.44, respectively. 


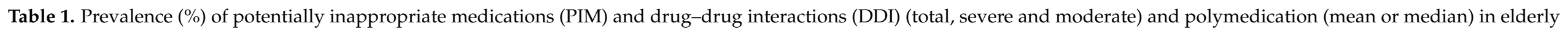

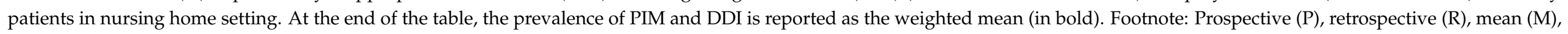
median (m), threshold (t), severe (S), moderate (M), not apply (NA), not determined (ND).

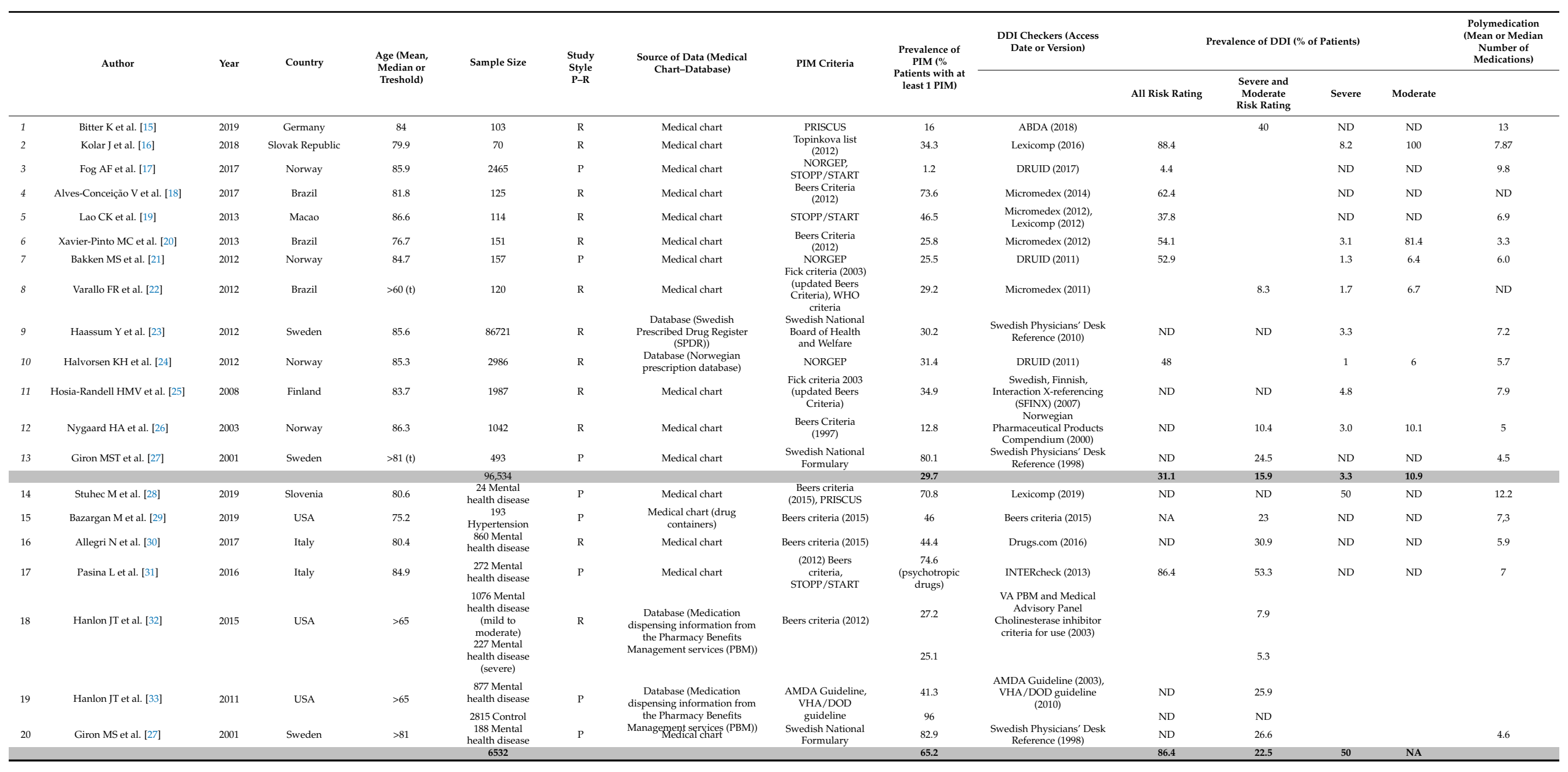




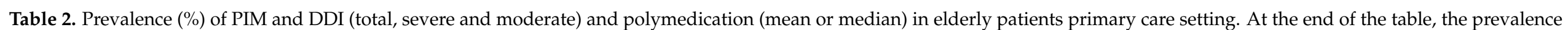

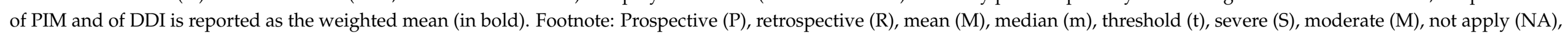
not determined (ND).

\begin{tabular}{|c|c|c|c|c|c|c|c|c|c|c|c|c|c|c|c|}
\hline & \multirow{2}{*}{ Author } & \multirow{2}{*}{ Year } & \multirow{2}{*}{ Country } & \multirow{2}{*}{$\begin{array}{l}\text { Age (Mean, } \\
\text { Median or } \\
\text { Treshold) }\end{array}$} & \multirow{2}{*}{ Sample Size } & \multirow{2}{*}{$\begin{array}{l}\text { Study } \\
\text { Style } \\
\text { P-R }\end{array}$} & \multirow{2}{*}{$\begin{array}{l}\text { Source of Data (Medical } \\
\text { Chart-Database) }\end{array}$} & \multirow{2}{*}{ PIM Criteria } & \multirow{2}{*}{$\begin{array}{l}\text { Prevalence of } \\
\text { PIM (\% } \\
\text { Patients with at } \\
\text { least } 1 \text { PIM) }\end{array}$} & \multirow[t]{2}{*}{$\begin{array}{l}\text { DDI Checkers (Access } \\
\text { Date or Version) }\end{array}$} & \multicolumn{4}{|c|}{ Prevalence of DDI (\% of Patients) } & \multirow{2}{*}{$\begin{array}{c}\text { Polymedication } \\
\text { (Mean or Median } \\
\text { Number of } \\
\text { Medications) } \\
\end{array}$} \\
\hline & & & & & & & & & & & All Risk Rating & $\begin{array}{c}\text { Severe and } \\
\text { Moderate } \\
\text { Risk Rating }\end{array}$ & Severe & Moderate & \\
\hline 1 & Bobrova et al. [34] & 2019 & Finland & 84.6 & 208 & $\mathrm{R}$ & $\begin{array}{l}\text { Database (PharmaService's } \\
\text { documentation system } \\
\text { ANJA) }\end{array}$ & EU []], PIM list & 73 & INXBASE (2017) & ND & ND & 2.4 & 50 & 5.9 \\
\hline 2 & Vatcharavongvan et al. [35] & 2019 & Thailand & $70.5(\mathrm{~m})$ & 400 & $\mathrm{R}$ & Medical chart & $\begin{array}{l}\text { Beers Criteria } \\
\text { (2015), } \\
\text { STOP/SART, } \\
\text { Winit-Watiana } \\
\text { Beers Criteria } \\
\text { (2015) } \\
\text { STOPP/START } \\
\text { Winit-Watiana }\end{array}$ & $\begin{array}{r}75.3 \\
\\
59 \\
40.3 \\
66.8\end{array}$ & 2015 Beers criteria & $\mathrm{NA}$ & 16 & $\mathrm{NA}$ & $\mathrm{NA}$ & $11(\mathrm{~m})$ \\
\hline 3 & Toivo et al. [36] & 2019 & Finland & $\begin{array}{l}81.6 \\
84.0\end{array}$ & $\begin{array}{l}65 \\
64\end{array}$ & $\mathrm{P}$ & Medical chart & Beers criteria (2015) & $\begin{array}{l}93.9 \\
90.6\end{array}$ & SFINX (2018) & $\begin{array}{l}\mathrm{ND} \\
\mathrm{ND}\end{array}$ & $\begin{array}{l}\mathrm{ND} \\
\mathrm{ND}\end{array}$ & $\begin{array}{l}10.8 \\
1.6\end{array}$ & $\begin{array}{l}\mathrm{ND} \\
\mathrm{ND}\end{array}$ & $\begin{array}{l}10.4 \\
9.8\end{array}$ \\
\hline 4 & Stuhec et al. [37] & 2019 & Slovenia & 77.5 & 91 & $\mathrm{R}$ & Medical chart & PRISCUS & $\mathrm{ND}$ & $\begin{array}{l}\text { Lexicomp (version } 4.0 .1 \\
\text { and } 4.0 .2 \text {. }\end{array}$ & ND & $\mathrm{ND}$ & 54.9 & ND & 13.8 \\
\hline 5 & Patel Ret al. [38] & 2018 & USA & $>65(t)$ & 703 & $\mathrm{P}$ & Medical chart & Beers criteria (2015) & 29 & $\begin{array}{l}\text { Beers criteria (2015) } \\
\text { Mcria }\end{array}$ & $\mathrm{NA}$ & 7.7 & $\mathrm{NA}$ & $\mathrm{NA}$ & 5.7 \\
\hline 6 & Novaes PH et al. [39] & 2017 & Brazil & $73.8(>60)$ & 368 & $\mathrm{R}$ & Medical chart & $\begin{array}{l}\text { Beers criteria (2012) } \\
\text { STOPP (2015) }\end{array}$ & $\begin{array}{l}42.1 \\
46.2\end{array}$ & $\begin{array}{l}\text { Medscape Drug } \\
\text { Interaction Checker (2016) }\end{array}$ & 72.3 & ND & 17.9 & ND & 4.46 \\
\hline 7 & Hanlon JT et al. [40] & 2017 & USA & 73.6 & 3055 & $\mathrm{R}$ & Database (Health ABC study) & Beers criteria (2015) & 34.0 & $\begin{array}{l}\text { Panel of } 70 \mathrm{DDI} \text { used for } \\
\text { the study (2017) }\end{array}$ & $\mathrm{ND}$ & 25.1 & $\mathrm{ND}$ & $\mathrm{ND}$ & $\mathrm{NA}$ \\
\hline 8 & Marin-Gorricho R et al. [41] & 2017 & Spain & 85.2 & 103 & $\mathrm{R}$ & Medical chart & $\begin{array}{c}\text { STOPP/START } \\
(2014)\end{array}$ & 81.6 & CheckTheMeds (2017) & 57.3 & $\mathrm{ND}$ & $\mathrm{ND}$ & ND & 7.4 \\
\hline 9 & Bazargan M et al. [42] & 2016 & USA & 73.5 & 400 & $\mathrm{R}$ & Interview & Beers criteria (2012) & 69.5 & $\begin{array}{l}\text { Healthline erug } \\
\text { interaction checker (2015) }\end{array}$ & ND & ND & 52.75 & ND & $\mathrm{NA}$ \\
\hline 10 & Popovic Bet al. [43] & 2014 & Croatia & 77 & 29418 & $\mathrm{R}$ & $\begin{array}{l}\text { Database (Croatian Health } \\
\text { Insurance Fund database) }\end{array}$ & $\begin{array}{l}\text { Mimica Matanović } \\
\text { and } \\
\text { Vlahof-Palčevsski } \\
(2012) \\
\text { (2012) }\end{array}$ & 62.4 & $\begin{array}{l}\text { Panel of } 49 \text { potentially } \\
\text { serious DDI used for the } \\
\text { study (2014) }\end{array}$ & $\mathrm{ND}$ & ND & ND & ND & $\mathrm{NA}$ \\
\hline 11 & Tora $\mathrm{H}$ et al. $[44]$ & 2014 & Sweden & 75.8 & 180059 & $\mathrm{R}$ & $\begin{array}{l}\text { Database (Swedish national } \\
\text { prescription repository) }\end{array}$ & $\begin{array}{l}\text { Swedish National } \\
\text { Board of Health } \\
\text { and Welfare }\end{array}$ & 30.8 & $\begin{array}{l}\text { First Databank's DDI } \\
\text { module (2013) }\end{array}$ & ND & 38.6 & ND & ND & 10.4 \\
\hline 12 & Steinman MA et al. [45] & 2014 & USA & 75 & 462405 & $\mathrm{R}$ & $\begin{array}{c}\text { Database (Merged VA } \\
\text { database-Medicare Program) }\end{array}$ & $\begin{array}{l}\text { Beers criteria (2012) } \\
\text { Zhan criteria }\end{array}$ & 26.0 & Lexicomp (2013) & ND & 30.2 & 4.4 & 30 & $\mathrm{NA}$ \\
\hline 13 & Lund BC et al. [46] & 2013 & USA & 75.8 & 1549824 & $\mathrm{R}$ & Database (VA database) & $\begin{array}{l}\text { Zhana criteriai } \\
\text { (adapterf from } 197 \\
\text { Beers criteria) } \\
\text { Fick criteria } 2003 \\
\text { (updated Beers } \\
\text { Criteria) }\end{array}$ & $\begin{array}{l}17.9 \\
16.5\end{array}$ & $\begin{array}{l}\text { Hansten PP, Horn J. } \\
\text { Applied Therapeutics Inc. } \\
\text { (1998) }\end{array}$ & $\mathrm{NA}$ & 3.75 & $\mathrm{NA}$ & $\mathrm{NA}$ & 5.5 \\
\hline 14 & Koper D et al. [47] & 2013 & Austria & 76.4 & 169 & $\mathrm{P}$ & $\begin{array}{c}\text { medical chart } \\
\text { Database Swedish }\end{array}$ & $\begin{array}{c}\text { Pritring) } \\
\text { Swedis Uational }\end{array}$ & 37.3 & Lexicomp (2012) & $\mathrm{ND}$ & 59.2 & 2.4 & 58 & 9.1 \\
\hline 15 & Haasum Y et al. [23] & 2012 & Sweden & 75.6 & 1260843 & $\mathrm{R}$ & $\begin{array}{l}\text { Database (Swedish } \\
\text { Prescribed Drug Register } \\
\text { SPDR) }\end{array}$ & $\begin{array}{l}\text { Sweours National } \\
\text { Board of Health } \\
\text { and Welfare }\end{array}$ & 11.6 & $\begin{array}{l}\text { Swedish Physicians' Desk } \\
\text { Reference (2010) }\end{array}$ & ND & ND & 3.2 & ND & 4.3 \\
\hline 16 & Halvorsen KH et al. [24] & 2011 & Norway & 83.0 & 8268 & $\mathrm{R}$ & $\begin{array}{l}\text { Database (Norwegian } \\
\text { prescription database) }\end{array}$ & $\begin{array}{l}\text { NORGEP } \\
\text { NORP }\end{array}$ & 24.6 & DRUID (2011) & 57 & ND & 2 & 10 & 5.7 \\
\hline 17 & Ghadimi $\mathrm{H}$ et al. [48] & 2011 & Iran & 73.0 & 2041 & $\mathrm{R}$ & Medical chart & $\begin{array}{l}\text { Fick criteria } 2003 \\
\text { (updated Beers } \\
\text { Criteria) }\end{array}$ & 30.3 & $\begin{array}{l}\text { Swedish Physicians' Desk } \\
\text { Reference (2009) }\end{array}$ & ND & 14.3 & 2.4 & 13.1 & 4.4 \\
\hline 18 & Pozzi C et al. [49] & 2010 & Italy & 73.0 & 1022 & $\mathrm{R}$ & $\begin{array}{l}\text { Database (ICARe Dicomano } \\
\text { Study database) } \\
\text { Database (Swedish }\end{array}$ & $\begin{array}{l}\text { Beers Criteria } \\
\text { (1991) } \\
\text { Swedish }\end{array}$ & 9.0 & Micromedex (2009) & $\mathrm{ND}$ & ND & 7.2 & ND & 2.2 \\
\hline 19 & Johnell K et al. [50] & 2009 & Sweden & 81.0 & 645429 & $\mathrm{R}$ & $\begin{array}{l}\text { Prescribed Drug Register } \\
\text { SPDR) }\end{array}$ & $\begin{array}{l}\text { Nationealisoard of } \\
\text { Health and Welfare }\end{array}$ & 22.5 & $\begin{array}{l}\text { Swedish Physicians' Desk } \\
\text { Reference (2009) }\end{array}$ & $\mathrm{ND}$ & $\mathrm{ND}$ & 6.9 & $\mathrm{ND}$ & 6.8 \\
\hline
\end{tabular}


Table 2. Cont.

\begin{tabular}{|c|c|c|c|c|c|c|c|c|c|c|c|c|c|c|c|}
\hline & \multirow{2}{*}{ Author } & \multirow{2}{*}{ Year } & \multirow{2}{*}{ Country } & \multirow{2}{*}{$\begin{array}{l}\text { Age (Mean, } \\
\text { Median or } \\
\text { Treshold) }\end{array}$} & \multirow{2}{*}{ Sample Size } & \multirow{2}{*}{$\begin{array}{l}\text { Study } \\
\text { Style } \\
\text { P-R }\end{array}$} & \multirow{2}{*}{$\begin{array}{l}\text { Source of Data (Medical } \\
\text { Chart-Database) }\end{array}$} & \multirow{2}{*}{ PIM Criteria } & \multirow{2}{*}{$\begin{array}{l}\text { Prevalence of } \\
\text { PIM (\% } \\
\text { Patients with at } \\
\text { least } 1 \text { PIM) }\end{array}$} & \multirow[t]{2}{*}{$\begin{array}{l}\text { DDI Checkers (Access } \\
\text { Date or Version) }\end{array}$} & \multicolumn{4}{|c|}{ Prevalence of DDI (\% of Patients) } & \multirow{2}{*}{$\begin{array}{c}\text { Polymedication } \\
\text { (Mean or Median } \\
\text { Number of } \\
\text { Medications) } \\
\end{array}$} \\
\hline & & & & & & & & & & & All Risk Rating & $\begin{array}{l}\text { Severe and } \\
\text { Moderate } \\
\text { Risk Rating }\end{array}$ & Severe & Moderate & \\
\hline 20 & Chrischilles EA et al. [51] & 2009 & USA & $>68(\mathrm{t})$ & 626 & $\mathrm{P}$ & $\begin{array}{l}\text { Database (Iowa medicare } \\
\text { beneficiaries) }\end{array}$ & $\begin{array}{l}\text { Beers Criteria } \\
\quad(1997)\end{array}$ & 51.4 & $\begin{array}{l}\text { Hansten PD, Horn JR. } \\
\text { Applied Therapeutics Inc. } \\
(1998)\end{array}$ & NA & 5.8 & $\mathrm{NA}$ & $\mathrm{NA}$ & 8.5 \\
\hline 21 & Haider SI et al. [52] & 2009 & Sweden & 80.9 & 626258 & $\mathrm{R}$ & $\begin{array}{l}\text { Database (Swedish } \\
\text { Prescribed Drug Register } \\
\text { SPDR) }\end{array}$ & $\begin{array}{l}\text { Swedish National } \\
\text { Board of Health } \\
\text { and Welfare }\end{array}$ & 34.6 & $\begin{array}{l}\text { Swedish Physicians Desk } \\
\text { Reference (2007) }\end{array}$ & ND & 25 & $\mathrm{ND}$ & $\mathrm{ND}$ & 5.8 \\
\hline 22 & Lapi Fet al. [53] & 2009 & Italy & $\begin{array}{l}72.7 \\
77.7\end{array}$ & 568 & $\mathrm{R}$ & $\begin{array}{l}\text { Database (ICRRe Dicomano } \\
\text { study database) }\end{array}$ & $\begin{array}{l}\text { Beers Criteria } \\
\text { (1991) }\end{array}$ & $\begin{array}{l}9.1 \\
5.1\end{array}$ & Micromedex (2008) & $\begin{array}{l}\mathrm{ND} \\
\mathrm{ND}\end{array}$ & $\begin{array}{l}20.1 \\
30.5\end{array}$ & $\begin{array}{l}4.7 \\
5.6\end{array}$ & $\begin{array}{l}\mathrm{ND} \\
\mathrm{ND}\end{array}$ & $\begin{array}{l}\mathrm{NA} \\
\mathrm{NA}\end{array}$ \\
\hline & & & & & 606692 & & $\begin{array}{l}\text { Prescribed Drug Register } \\
\text { SPDRR } \\
\text { Database (Swedish }\end{array}$ & 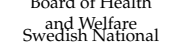 & 13.6 & & $\mathrm{ND}$ & ND & 3.7 & ND & 4.6 \\
\hline 24 & Johnell K et al. [55] & 2007 & Sweden & 82 & 732228 & $\mathrm{R}$ & $\begin{array}{l}\text { Prescribed Drug Register } \\
\text { SPDR) } \\
\text { Database (PRug subsidy }\end{array}$ & $\begin{array}{l}\text { Board of Health } \\
\text { and Welfare }\end{array}$ & 16.5 & $\begin{array}{l}\text { Swedish Physicianss ' Desk } \\
\text { Reference (2007) }\end{array}$ & ND & ND & 4.1 & ND & 5.4 \\
\hline 25 & Bregnhoj Let al. [56] & 2007 & Denmark & 76.6 & 212 & $\mathrm{R}$ & $\begin{array}{l}\text { Databasese( (rug gusbsidy } \\
\text { system of Danish } \\
\text { pharmacies) }\end{array}$ & MAI & 84 & Hansten and Horn (2004) & $\mathrm{NA}$ & 0.6 & $\mathrm{NA}$ & $\mathrm{NA}$ & 7.6 \\
\hline 26 & Cannon KT et al. [57] & 2006 & USA & 78 & 786 & $\mathrm{R}$ & Medical chart & Beers criteria (1997) & 31 & $\begin{array}{l}\text { Mutlidisisciplinary } \\
\text { Medication Management } \\
\text { (M3) Project criteria (2005) }\end{array}$ & $\mathrm{NA}$ & NA & 10 & $\mathrm{NA}$ & 8.0 \\
\hline 27 & Zhan C et al. [58] & 2005 & USA & $>65(t)$ & 70203 & $\mathrm{R}$ & $\begin{array}{l}\text { Database (National } \\
\text { Ambulatory Medical Care } \\
\text { Survey NAMCS) }\end{array}$ & $\begin{array}{l}1997 \text { Beers criteria, } \\
\text { Mac Leod 1997, } \\
\text { Panel expert } 50 \\
\text { drug-diseasese } \\
\text { combinations }\end{array}$ & 2.58 & $\begin{array}{l}1997 \text { Beers criteria, Mac } \\
\text { Leed 19977 Panel expert } 6 \\
\text { drug-drug combinations }\end{array}$ & $\mathrm{NA}$ & 0.76 & $\mathrm{NA}$ & $\mathrm{NA}$ & $\mathrm{NA}$ \\
\hline 28 & Azoulay Let al. [59] & 2003 & Iran & 72.6 & 3000 & $\mathrm{R}$ & Medical chart & Beers criteria (1997) & 27.6 & $\begin{array}{l}\text { Micromedex (Drug-Reax } \\
\text { vol 114) }\end{array}$ & 9.5 & $\mathrm{ND}$ & ND & $\mathrm{ND}$ & 3.7 \\
\hline 30 & Schmader K et al. [61] & 1994 & USA & 69.8 & 208 & $\mathrm{R}$ & Medical chart & MAI & $\begin{array}{l}14 \\
14\end{array}$ & MAI (1994) & NA & $\begin{array}{c}2.4 \\
0\end{array}$ & $\mathrm{NA}$ & $\begin{array}{l}\mathrm{NA} \\
\mathrm{NA}\end{array}$ & NA \\
\hline 31 & Courlet P [62] & 2019 & Switzerland & 71 & $\begin{array}{l}7,872,649 \\
122 \mathrm{HIV}\end{array}$ & $\mathrm{P}$ & $\begin{array}{l}\text { Database (Swiss HIV cohort } \\
\text { study) }\end{array}$ & Beers criteria (2015) & $\begin{array}{c}19.1 \\
31\end{array}$ & $\begin{array}{l}\text { University of Liverpool } \\
\text { drug interaction checker, } \\
\text { Up-to-Date database }\end{array}$ & $\begin{array}{c}45.3 \\
11\end{array}$ & $\begin{array}{l}14.65 \\
\mathrm{ND}\end{array}$ & $\begin{array}{l}4.4 \\
\mathrm{ND}\end{array}$ & $\begin{array}{l}29.6 \\
\mathrm{ND}\end{array}$ & 4 \\
\hline 32 & Lund BC et al. [63] & 2017 & USA & $>66$ & 19318 Cancer & $\mathrm{R}$ & Database (SEER Medicare) & Beers criteria (2012) & 37.3 & $\begin{array}{l}\text { Micromedex (2017) } \\
\text { Norwegian drug }\end{array}$ & & & 5 & 3.2 & \\
\hline 33 & Oesterhus R et al. [64] & 2017 & Norway & 77 & $\begin{array}{l}251 \text { Mental } \\
\text { health disease }\end{array}$ & $\mathrm{R}$ & Database (DemWest study) & NORGEP & 14 & $\begin{array}{l}\text { interaction database (2014, } \\
\text { Norwegian medicines } \\
\text { agency) }\end{array}$ & 41 & & 1.6 & 36 & 4 \\
\hline 34 & Wucherer D et al. [65] & 2017 & Germany & 79.8 & $\begin{array}{l}446 \text { Mental } \\
\text { health disease }\end{array}$ & $\mathrm{R}$ & $\begin{array}{l}\text { Database (Dementiaa } \\
\text { DelpHi-MY study) }\end{array}$ & PRISCUS & 22.9 & ABDA (2017) & $\mathrm{ND}$ & $\mathrm{ND}$ & 3.8 & 34.8 & 6.4 \\
\hline 35 & Yazdanshenas $\mathrm{H}$ et al. [66] & 2016 & USA & $>65$ & $\begin{array}{l}187 \text { Pain } \\
1875\end{array}$ & $\mathrm{R}$ & $\begin{array}{l}\text { Medical chart } \\
\text { Merlo }\end{array}$ & Beers criteria (2012) & 83 & Healthline (2015) & 60 & $\mathrm{ND}$ & ND & ND & \\
\hline 36 & Suehs B.T. et al. [67] & 2016 & USA & $>65$ & $\begin{array}{l}66275 \\
\text { Overactive } \\
\text { bladder }\end{array}$ & $\mathrm{R}$ & Medical chart & Beers criteria (2012) & 31.1 & Beers criteria (2012) & & $\begin{array}{l}14.3 \\
\text { (anticholinergic } \\
\text { DDI) }\end{array}$ & & & \\
\hline 37 & Dionne PA et al. [68] & 2013 & Canada & 73.8 & $\begin{array}{l}744 \text { Overactive } \\
\text { bladder }\end{array}$ & $\mathrm{R}$ & Database (ESA survey) & $\begin{array}{l}\text { Fick criteria 2003 } \\
\text { (updated Beers } \\
\text { Criteria) }\end{array}$ & 22 & Micromedex (2012) & 15 & ND & ND & ND & \\
\hline 38 & Gallagher [69] & 2001 & USA & 88 & $\begin{array}{l}146 \text { Heart } \\
\text { Failure }\end{array}$ & $\mathrm{R}$ & Medical chart & Beers criteria (1997) & 8.2 & $\begin{array}{l}\text { Panel of DDI used for the } \\
\text { study (1999) }\end{array}$ & $\mathrm{ND}$ & 44.5 & $\mathrm{ND}$ & $\mathrm{ND}$ & 8 \\
\hline
\end{tabular}




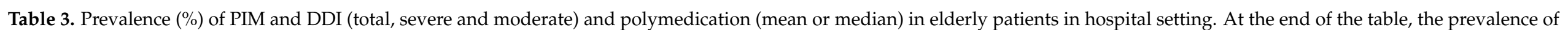

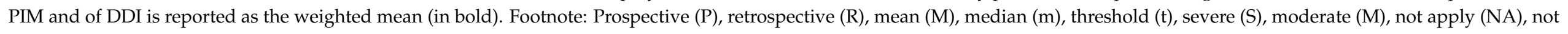
determined (ND).

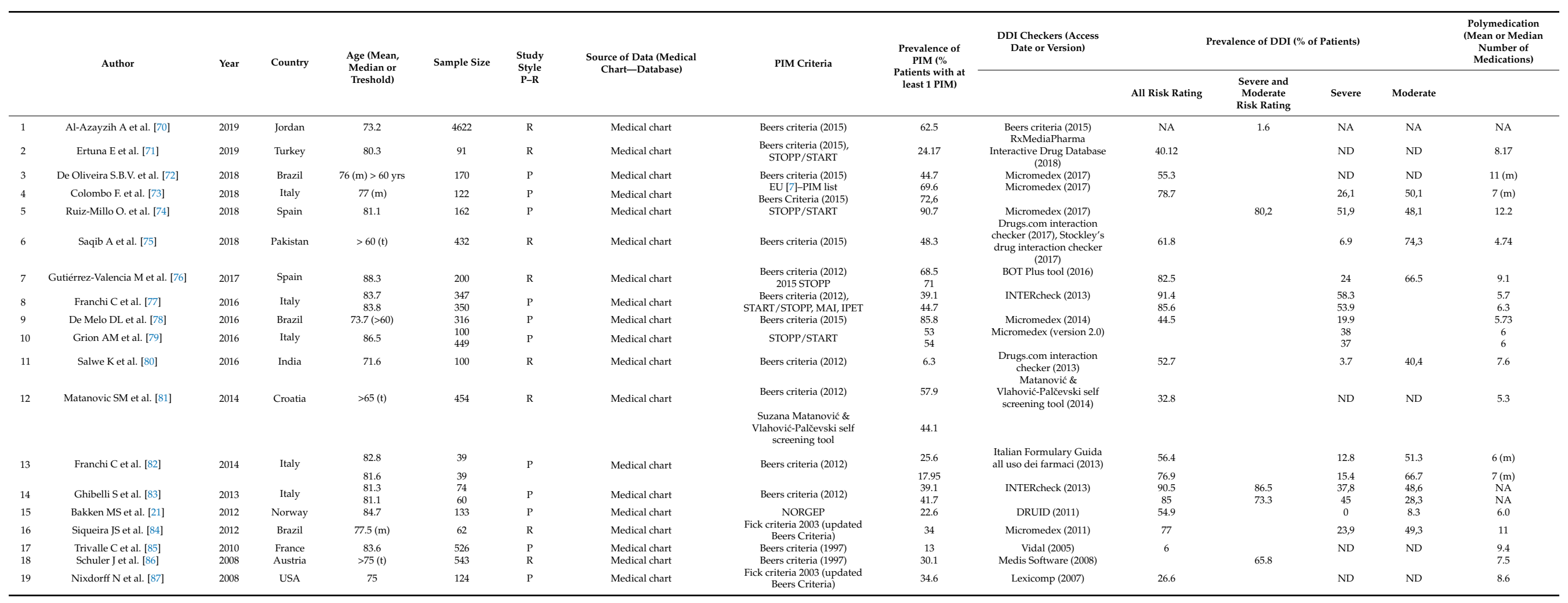


Table 3. Cont.

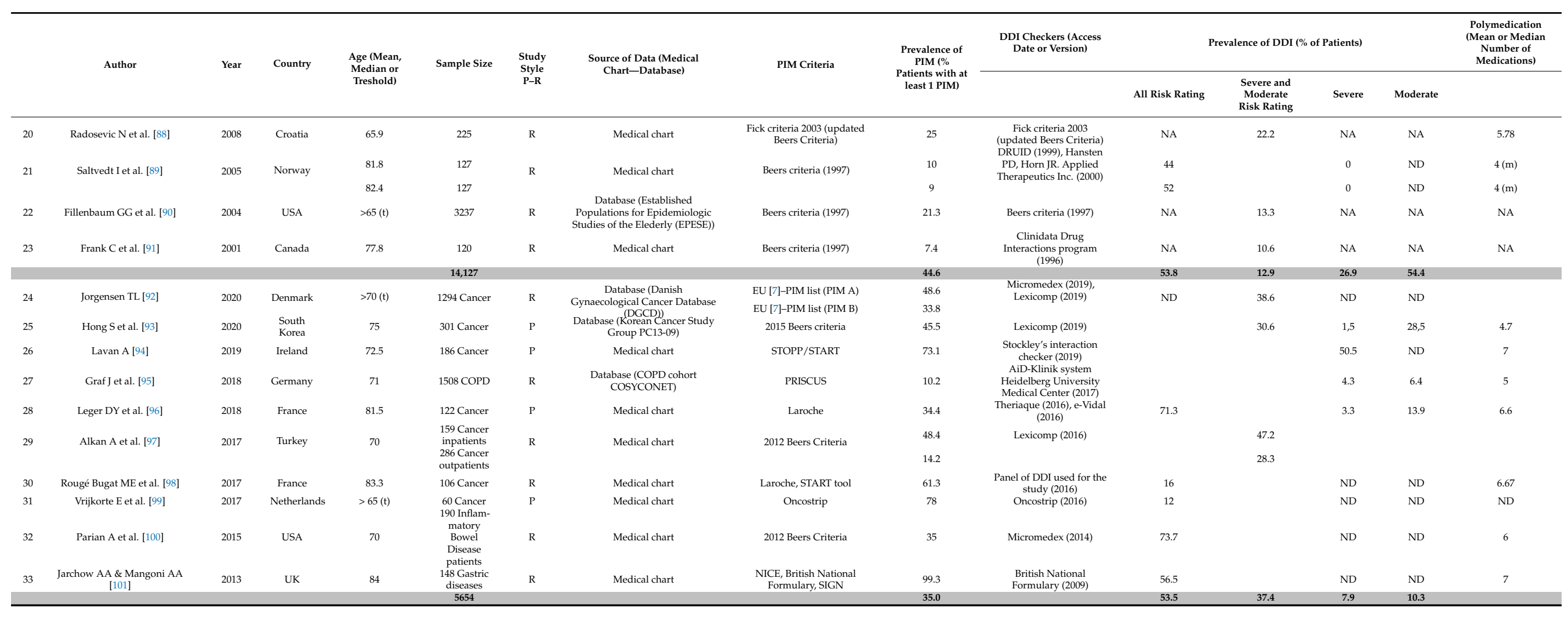


Table 4. Mapping of the potential risk rating classification of DDI among checker tools used in the studies referenced in the current systematic review.

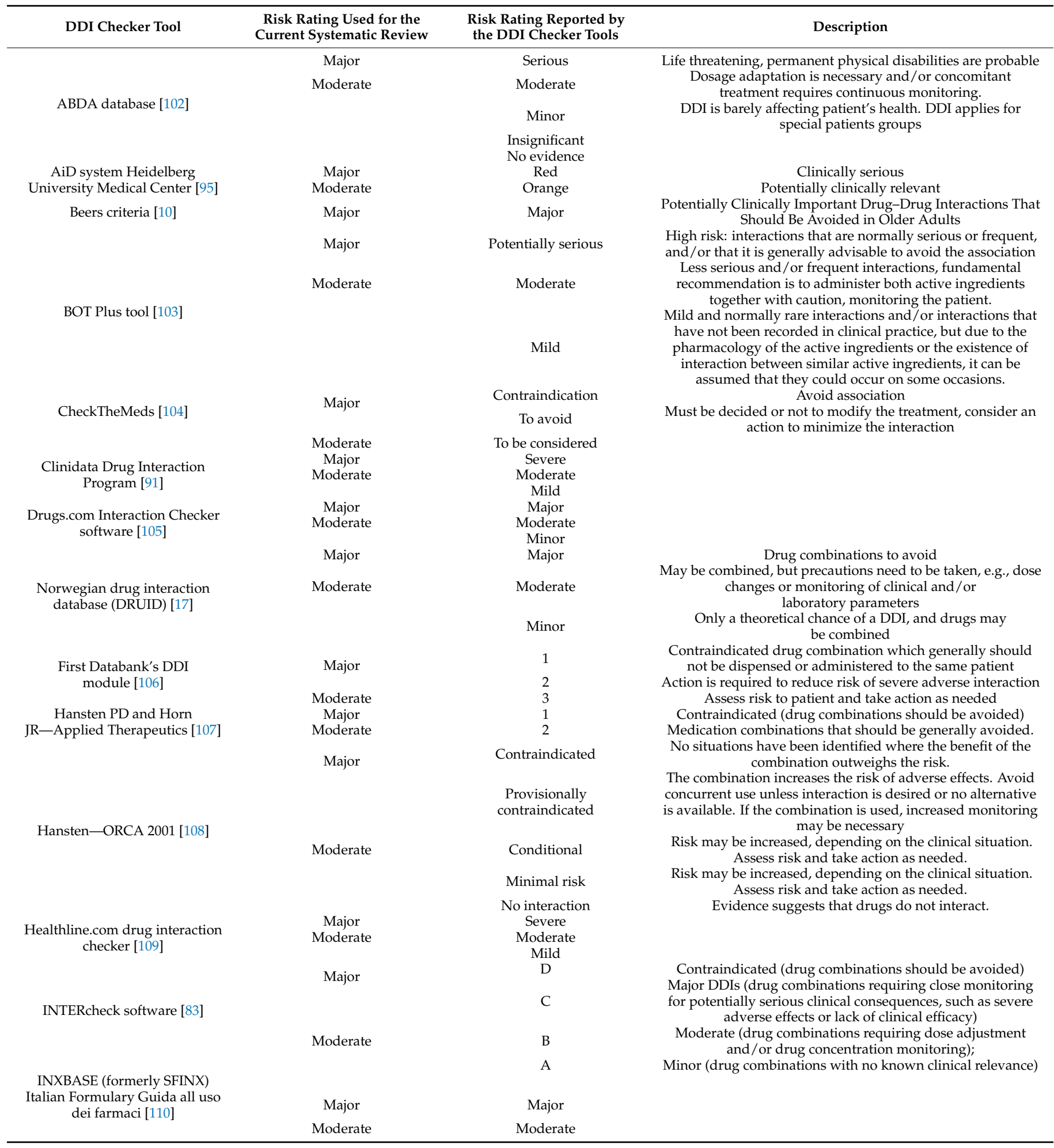


Table 4. Cont.

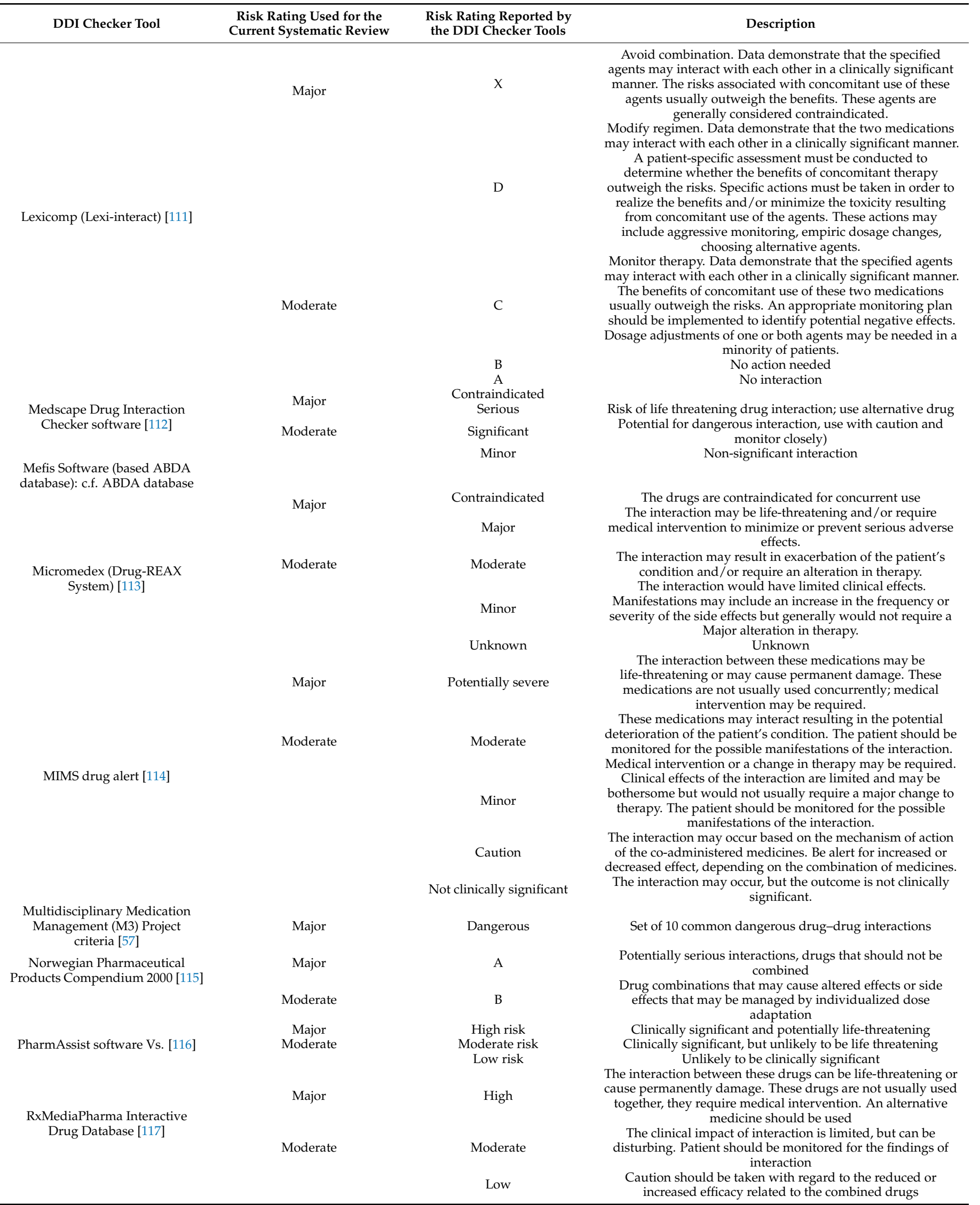


Table 4. Cont.

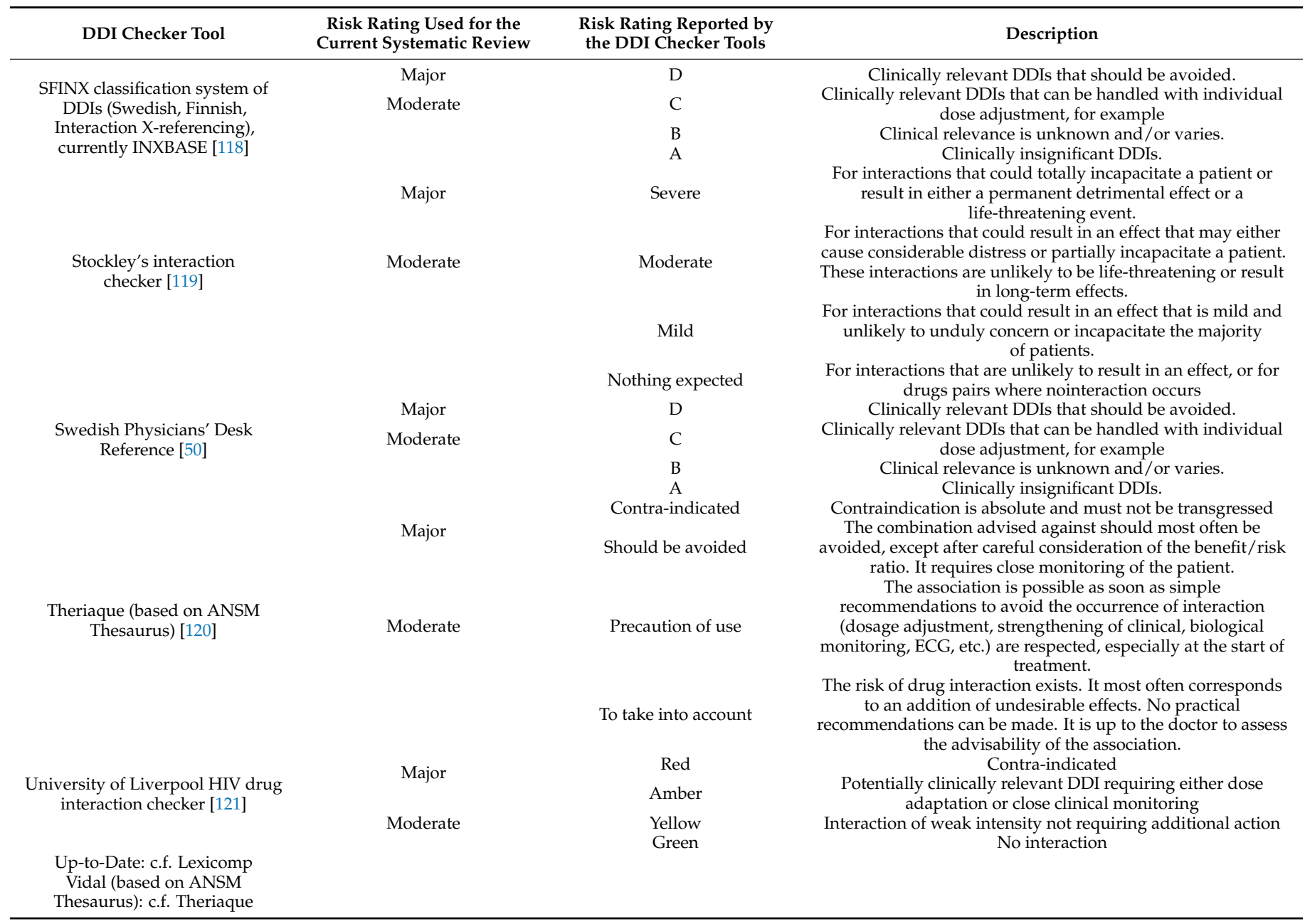

\subsection{Drug-Drug Interactions}

Our mapping of the different risk rating classification provided by the checker tools allowed us to report only clinically relevant DDI, i.e., considered as major and moderate risk (Table 4).

We checked for a potential difference between the tools used to identify DDI by comparing Micromedex, Lexicomp, DRUID, Swedish Physician's Desk reference and Intercheck that were the most used tools to identify DDI (in 20\%, 7\%, 7\%, 13\% and 6\% of the studies, respectively). The average prevalence of DDI reported by using these tools were $19.9 \%, 17.5 \%, 1.1 \%, 4.6 \%$ and $48.8 \%$, respectively for severe DDI, and $47.1 \%$, $62.7 \%, 7.7 \%, 13.1 \%$ and $38.4 \%$ for moderate DDI. Without consideration of settings, the distribution was normal $(p<0.001)$ for severe DDI; and a significant difference between the tools $(p=0.018)$ in their ability to detect severe DDIs was found. Concerning moderate DDI, there was a normal distribution $(p=0.057)$ but no significant difference between the tools $(p=0.077)$.

Since Micromedex was the most used tool in the general population $(n=14,20 \%)$, we made a comparison of DDI (severe and moderate) according to Micromedex between the settings. The prevalence of severe DDI was 6.1\% (95\% CI: $4.5-7.7), 2.5 \%$ (95\% CI: $1.1-3.8)$ and $32.8 \%$ (95\% CI: 23.0-42.7) in primary care, nursing home and hospital revealing differences between settings $(p<0.001)$. Concerning moderate DDI, a comparison between the three settings was not possible given that Micromedex was not used to identify such DDI in primary care. However, no difference between nursing home and hospital was 
found ( $p=0.057)$. Indeed, moderate DDI prevalence was 48.3\% (95\% CI $-23.9-120.6)$ and $49 \%$ (95\% CI 47.6-50.4) for these settings.

The weighted mean prevalence of severe DDI (Figure 1) was in the rank order: hospital $28.9 \%$ (95\% CI: $17.2-40.6)$, primary care $4.4 \%$ (95\% CI 3.2-5.6) and nursing home $3.3 \%$ (95\% CI: 3.1-3.4). The same rank order was found for moderate DDI with $54.4 \%$ (95\% CI $38.8-70.0)$ in hospital, $29.6 \%$ (95\% CI: 28.5-30.6) in primary care and $10.9 \%(95 \%$ CI $2.3-19.6)$ in nursing home, respectively.
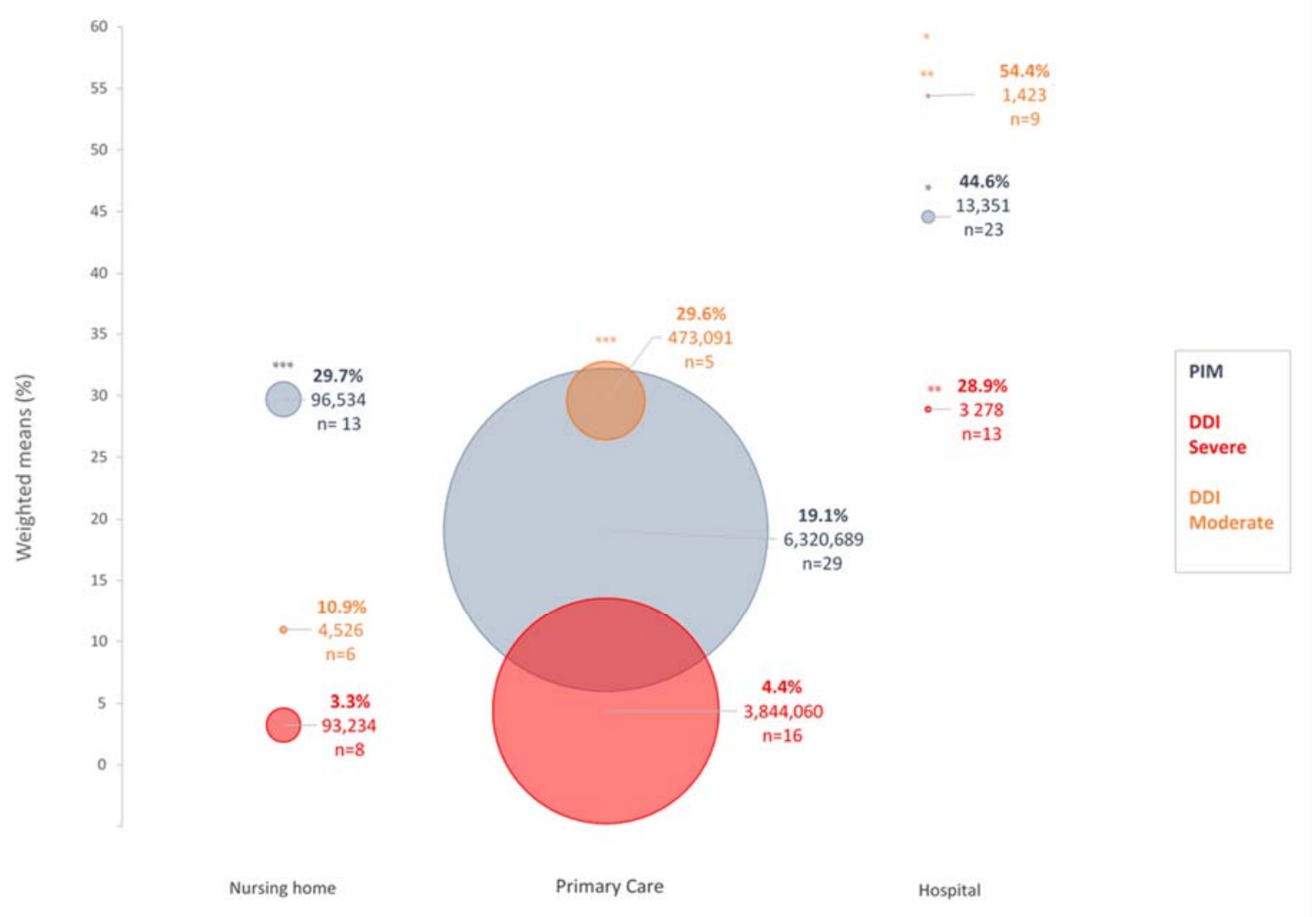

Figure 1. Bubble plot of the prevalence (\%) of potentially inappropriate medication (PIM) (grey) and of severe (red) and moderate (orange) drug-drug interaction (DDI) in elderly patients in primary care, nursing home and hospital settings. The prevalence of both PIM and DDI has been reported as a weighed mean. The surface of the circle is proportional to the total sample size of the studies used in the calculation of the parameters (the sample size, the weighed mean and the number of studies used are reported). *: significant difference between hospital and primary care settings. ${ }^{* *}$ : significant difference between hospital and nursing home settings. ${ }^{* *}$ : significant difference between primary care and nursing home settings.

Severe and moderate DDI displayed significant differences between settings $(p<0.001$ for each). Using pairwise comparisons, no significant differences were noticed for severe DDI between nursing home and primary care $(p=0.21)$ nor between primary care and hospital $(p=0.054)$. However, the prevalence of severe and moderate DDI between nursing home and hospital was significantly different $(p<0.001$ and $p=0.0035$ respectively). Differences in the prevalence of moderate DDI between primary care and hospital $(p=0.0035)$ and between primary care and nursing home $(p<0.001)$ were also observed.

The prevalence in DDI in specific populations of patients and in the different settings is presented in Tables 1-3.

\subsection{Prevalence of Drug-Drug Interactions in the Clinical Data Warehouse}

DDI with the 15 selected pairs (DDI as victim drug and inhibitor) occurred in 769 patients of our sample of 8434 patients leading to a global prevalence of $9.1 \%$ (Table 5). 
The prevalence of DDI for these selected pairs ranged from 0.1\% (CYP2D6 inhibition of tramadol metabolism by terbinafine) to $19.4 \%$ (P-gp transport inhibition of apixaban by amiodarone).

Table 5. Prevalence of DDI of moderate or major intensity (by inhibition of a metabolic or of a transport pathway) regarding six selected PIM in hospitalized patients. Data were extracted from the clinical data warehouse of Rennes University Hospital (eHop). DDI were checked by using Micromedex and literature search in Pubmed. Specific bibliographic references were added when DDI was not indexed in Micromedex. Footnote: not indexed (-).

\begin{tabular}{|c|c|c|c|c|c|c|c|c|}
\hline PIM & $\begin{array}{c}\text { Patient } \\
\text { Sample Size }\end{array}$ & $\begin{array}{c}\text { CYP and/or } \\
\text { Transporter Pathway }\end{array}$ & Inhibitors & $\begin{array}{l}\text { Patients with } \\
\text { DDI }\end{array}$ & Prevalence $(\%)$ & $\begin{array}{l}\text { Risk Rating from } \\
\text { Micromedex }\end{array}$ & Mechanism & Effects \\
\hline \multirow{5}{*}{ Tramadol } & \multirow{5}{*}{5896} & \multirow{5}{*}{ 2D6 } & Fluoxetine & 69 & $1.17 \%$ & \multirow{5}{*}{$\begin{array}{c}\text { Major } \\
- \\
-\end{array}$} & Inhibition of & \multirow{5}{*}{$\begin{array}{c}\text { Increased tramadol exposure and reduced } \\
\text { concentrations of the active metabolite (risk } \\
\text { of signs and symptoms of opioid } \\
\text { withdrawal, reduction of efficacy and } \\
\text { serotonin syndrome) }\end{array}$} \\
\hline & & & Paroxetine & 168 & $2.85 \%$ & & CYP2D6-mediated & \\
\hline & & & Terbinafine & 6 & $0.10 \%$ & & tramadol metabolism & \\
\hline & & & Posaconazole & 2 & $0.10 \%$ & & CYP3A4 inhibition & \\
\hline & & & Voriconazole & 4 & $0.21 \%$ & & CYP3A4 inhibition & \\
\hline \multirow[t]{3}{*}{ Apixaban } & \multirow{3}{*}{1922} & \multirow{3}{*}{ 3A4/P-gp } & Clarithromycin & 20 & $1.04 \%$ & - & CYP3A4 inhibition and & \multirow{3}{*}{ Bleeding risk [122] } \\
\hline & & & Verapamil & 46 & $2.39 \%$ & . & $\begin{array}{l}\text { P-gp inhibition } \\
\text { P-gp competition }\end{array}$ & \\
\hline & & & Amiodarone & 366 & $19.04 \%$ & - & P-gp competition & \\
\hline \multirow{3}{*}{ Digoxin } & \multirow{3}{*}{345} & \multirow{3}{*}{ P-gp } & Verapamil & 11 & $3.19 \%$ & Major & $\begin{array}{l}\text { Inhibition of renal and /or } \\
\text { extrarenal clearance, } \\
\text { additive effects on } \mathrm{AV} \\
\text { node conduction } \\
\text { Inhibition of }\end{array}$ & $\begin{array}{l}\text { Increased serum digoxin concentrations } \\
\text { and risk of digitalis toxicity and complete } \\
\text { heart block }\end{array}$ \\
\hline & & & Clarithromycin & 3 & $0.87 \%$ & Major & $\begin{array}{l}\text { P-glycoprotein-mediated } \\
\text { digoxin efflux transport by } \\
\text { clarithromycin }\end{array}$ & $\begin{array}{l}\text { Increased risk of digoxin toxicity (nausea, } \\
\text { vomiting, arrhythmias) }\end{array}$ \\
\hline & & & Amiodarone & 60 & $17.39 \%$ & Major & $\begin{array}{l}\text { Inhibition of } \\
\text { p-glycoprotein by } \\
\text { amiodarone, and reduction } \\
\text { of digoxin clearance; } \\
\text { interference with } \\
\text { amiodarone by digoxin } \\
\text { Inhibition of }\end{array}$ & $\begin{array}{l}\text { Increased risk of digoxin toxicity (nausea, } \\
\text { vomiting, arrhythmias) and potentiated } \\
\text { effects of amiodarone (bradycardia, sinus } \\
\text { arrest, AV block). Drug interactions may } \\
\text { persist for weeks to months after } \\
\text { amiodarone discontinuation. }\end{array}$ \\
\hline Clozapine & 116 & $1 \mathrm{~A} 2$ & Ciprofloxacin & 1 & $0.86 \%$ & Major & $\begin{array}{l}\text { CYP1A2-mediated } \\
\text { clozapine metabolism and } \\
\text { additive QT-interval } \\
\text { prolongation } \\
\text { Inhibition of }\end{array}$ & $\begin{array}{l}\text { Increased clozapine exposure and risk of } \\
\text { QT prolongation }\end{array}$ \\
\hline \multirow[t]{2}{*}{ Glimepiride } & \multirow[t]{2}{*}{99} & \multirow[t]{2}{*}{$2 \mathrm{C} 9$} & Amiodarone & 10 & $10.10 \%$ & Moderate & $\begin{array}{l}\text { CYP2C9-mediated } \\
\text { glimepiride metabolism by } \\
\text { amiodarone } \\
\text { Inhibition of }\end{array}$ & Increased plasma levels of glimepiride \\
\hline & & & Fluconazole & 2 & $2.02 \%$ & - & $\begin{array}{l}\text { CYP2C9-mediated } \\
\text { glimepiride metabolism by } \\
\text { amiodarone } \\
\text { Inhibition of }\end{array}$ & Risk of hypoglycemia [123] \\
\hline Quetiapine & 56 & $3 \mathrm{~A} 4$ & Clarithromycin & 1 & $1.79 \%$ & Major & $\begin{array}{l}\text { CYP3A4-mediated } \\
\text { metabolism of quetiapine; } \\
\text { additive effects on } \\
\text { QT-interval prolongation }\end{array}$ & $\begin{array}{l}\text { Increased quetiapine exposure and } \\
\text { increased risk of QT prolongation }\end{array}$ \\
\hline
\end{tabular}

\section{Discussion}

This systematic review showed that the mean weighted prevalence of PIM was in the following rank order: hospital $>$ nursing home $>$ primary care with significant differences between these settings (Figure 1). The prevalence of severe and moderate risk DDI in hospital settings was higher than those in primary care and nursing home (Figure 1), and there were few studies dealing with DDI involving specifically PIM.

\subsection{Potentially Inappropriate Medications}

The polymedication rate was similar between settings, and cannot be accounted for in the differences in PIM prevalence. When considering only studies using Beers criteria, the prevalence of PIM remained the highest in the hospital setting but prevalence in primary care setting exceeded the one in nursing home setting. The highest prevalence of PIM in the hospital setting may be related to the more severe conditions of patients and to the fact that some PIM are due to drug-disease and drug-syndrome interactions (e.g., heart failure, delirium, late stage of chronic kidney disease). Moreover, as recently suggested, hospital admission per se (as a result of change in patient clinical status and of intensification of healthcare) was shown to be an important driver of PIM independently of polymedication and multimorbidity [124].

However, differences in PIM prevalence among settings may be somewhat biased by the tools used that have different criteria of detection. Indeed, only a few medications are common to all the PIM's criteria, resulting from differences in drug dosage as well as market availability [125]. If Beers criteria were the most used tool in the reported studies, NORGEP and STOPP/START were the two other most used tools. The higher prevalence observed 
with Beers criteria (38.6\%), compared to NORGEP (26.0\%) may be related to the fact that NORGEP criteria do not include medications that would be considered inappropriate for specific co-morbidities and hence led to an underestimation of the prevalence. On the other hand, the higher prevalence reported with STOPP/START $(60.4 \%)$ may result from the fact that this tool identifies underuse and overuse of medications [126]. Hence, the prevalence of PIM may be dependent on the tools used in the studies.

\subsection{Drug-Drug Interactions}

We decided to focus only on clinically relevant DDI (i.e., considered as major and moderate risk) that are highly likely to alter the effectiveness or toxicity of one or more medications. Clinically relevant DDI can lead to measurable patient adverse events taking into account an individual patient's profile. However, there are very few studies considering DDI resulting in actual harm to the patients.

Reporting only clinically relevant DDI was made possible by a mapping of the different risk rating classification provided by the checker tools that we performed (Table 4). Besides medication severity classification, differences in DDI checker tools also result from differences in the knowledge databases they are based on. A comparison of three commercial DDI knowledge databases showed significant differences in their numbers of clinical medication pairs and a limited overlap [127]. In a data mining study performed in 10,506 elderly patients' treated statins, we showed differences in the risk rating, and in the individuals DDI among the different statins used [128].

The higher prevalence of severe and moderate risk DDI in hospital settings (Figure 1) compared to primary care and nursing home is not unlikely given the nature of medication treatments that are prescribed to hospitalized patients as a result of more severe conditions. This higher prevalence in hospital setting was apparently not related to the polymedication rate that was very similar among the different settings.

In a recent systematic review from studies achieved in hospitalized patients [129], the rate of potential DDI (all risk rating) was estimated as 33\%, with a rather large variability (95\% CI: 17.3-51.3). Our estimation of clinically relevant DDI in elderly patients hospitalized from general population displayed a higher load of DDI since the prevalence of severe and moderate DDI averaged $28.9 \%$ and $54.4 \%$, respectively.

The fact that a rather high prevalence of clinically relevant DDI occurs in a population with a high prevalence of PIM prescription (around half of the population) is of particular concern. Indeed, DDI can occur with a PIM as the victim medication, and may enhance their inappropriate character by increasing their unfavorable balance of benefits and harm. Indeed, the enzymatic inhibition of the metabolism of a PIM may enhance its systemic exposure and, by the way, may increase either its clinical effects and/or its side effects. However, the clinical impact of the increase in the inappropriate character of PIM depends on the magnitude of the increase in systemic exposure as well as on the therapeutic index of the PIM. PIM generating DDI (i.e., as a perpetrator medication) is another concern to investigate. Even though the DDI will not modify per se the inappropriate character of the PIM, it could potentially have deleterious effects related to the induction or the inhibition of the metabolic pathway and/or transporter involved in the disposition of the medication undergoing DDI (i.e., victim medication).

This review clearly showed that the issue of DDI occurring specifically with PIM has not yet been of concern. Indeed, only 4 studies out of 91 have considered this issue. In a recent study involving 368 patients in a primary care setting, the association between polypharmacy, PIM use and DDI (called "iatrogenic triad") was evidenced in around $30 \%$ of the patients. DDI and PIM were encountered in $8.2 \%$ of the patients [39]. In a study involving 1987 nursing home residents, 6.6\% of PIM users were susceptible to major DDI [25]. However, these studies did not report the prevalence of DDI involving specifically PIM.

More interestingly, a study involving 120 nursing home residents showed that potential DDI in PIM occurred in $18.3 \%$ of the prescriptions with $60 \%$ of these DDI considered as 
potentially harmful [22]. In a study focusing on the use of benzodiazepine in 744 primary care patients, the prevalence of DDI in benzodiazepines considered as PIM occurred in $7 \%[68]$.

Ideally, in elderly patients, PIMs should always be reviewed, and DDI should be always checked. However, with regard to DDI, attention should be paid to those occurring with PIM since they could increase their inappropriate character. Even though this issue should be tackled in priority in the hospital setting, it is also relevant for patients in primary care and nursing homes even if the prevalence of clinically relevant DDI was lower, especially in nursing homes (Figure 1).

Figure 1 also suggests that studies in the hospital setting involving a higher number of patients are warranted to get a better picture of DDI in PIM in this setting. If it could be difficult to reach the sample sizes reported in primary care studies, the development of prescriptome analytics from clinical data-warehouses should help in reaching this goal [130].

An overview of the future management of the burden of DDI of the healthcare system is beyond the scope of this paper, but we think that attention should be paid to DDI in PIM in elderly people given the epidemiologic transition characterized by the population aging and the emergence of chronic diseases requiring long-term medication treatment with medications classified as PIM [10]. In the hospital setting, DDIs are estimated to contribute to $3-5 \%$ of adverse events. They are also a significant cause of hospitalization (either via emergency department visits or via hospital admissions). In a retrospective study on the origin of hospital admissions, adverse drug reactions (ADRs) were rated as a significant cause and around $25 \%$ of these ADRs were related to a DDI [131].

Given the paucity of data on DDI occurring specifically with PIM in the literature mentioned above, we have performed during the course of this review a preliminary study by checking 15 DDIs involving 6 PIMs as victim medications involving 8464 elderly patients in our hospital. The global prevalence of clinically relevant DDI (i.e., 9.1\%) observed in our sample should be considered with caution because only 15 potential DDIs were checked, and it is thus a real underestimation of the true prevalence. However, this short survey of a subset of 15 DDIs of moderate or major risk regarding only 6 PIMs suggests that a comprehensive investigation of DDI involving PIM should implemented. This could be facilitated by using an automatic DDI identification as we previously set up for a study on DDI with statins [128].

Furthermore, considering that some of these DDIs were risk-rated as major (Table 5), a further study would ideally investigate the potential negative outcomes of DDI in PIM on ADR, and their consequences (hospitalization as well as morbidity and mortality).

\subsection{Strength and Limitations}

This systematic review has several strengths. First, our study included a broad range of studies encompassing the primary care, nursing home and hospital settings. Second, given the fact that different DDI checker software are used worldwide with different classifications of risk ratings, we have performed a mapping of the DDI risk rating to allow a better classification of clinically significant DDIs, and of the estimation of their prevalence. Third, this is the first review of DDIs in elderly patients in whom PIMs are prescribed.

This study has several limitations. First, given the heterogeneity of the selected studies in terms of methodology and tools used as well as in reporting data, there are some missing data precluding a formal meta-analysis and its statistical evaluation. Nevertheless, weighted means in PIM and DDI prevalence allowed us to report some differences between settings. Second, we only performed the literature search using PubMed and Web-ofScience and did not identify grey literature so that some studies may have not been included. Third, the low number of studies in certain combinations (setting/PIM or setting/DDI) may contribute to some uncertainty in the point estimates and $95 \%$ CI. Fourth, the huge difference in the total sample size between settings (primary care $>>$ nursing home > hospital) may constitute a bias in comparison. 


\section{Conclusions: Future Research}

The current paper presents an in-depth review of the literature regarding the topic of DDI in elderly patients using PIM. It was performed with a mapping of DDI checker tools we thought to be a prerequisite for meaningful comparisons between studies. To our knowledge, this is the first systematic review of this issue.

We showed a higher prevalence of PIM, and of severe and moderate DDI, in the hospital setting compared to nursing home and primary care, unrelated to polymedication rate. Within the studies on DDI in elderly in whom PIM were prescribed, the issue of DDI occurring specifically with PIM has not yet been of concern, since only four studies involving a rather limited number of patients investigated this topic.

To help clinicians and pharmacists to identify at risk elderly patients, strategies to assess the appropriateness of medication use in elderly should additionally consider DDIs that specifically involve PIM. DDI software providers should implement alerts when a DDI occurs with PIM with a clinical decision support so that healthcare professionals can easily prevent and manage these DDIs. This would be most helpful in the hospital setting where PIMs and DDIs are highly prevalent.

Supplementary Materials: The following are available online at https://www.mdpi.com/1999 -4923/13/2/266/s1, Supplementary material 1: PRISMA checklist; Supplementary material 2. PRISMA flow chart of review screening, selection, exclusions and final included studies; Supplementary material 3: Pubmed literature search: Drug interactions [MeSH Terms] AND $(((()((()(\mathrm{In}-$ appropriate prescribing[MeSH Terms]) OR Inappropriate Medication[Title/Abstract]) OR Inappropriate Medications[Title/Abstract]) OR Inappropriate Prescription[Title/Abstract]) OR Inappropriate Prescriptions[Title/Abstract]) OR Inappropriate Prescribing[Title/Abstract]) OR Inappropriate Medicines[Title/Abstract]) OR Inappropriate Drugs[Title/Abstract]) OR Inappropriate Drug[Title/Abstract]) OR high-risk medications[Title/Abstract]) OR Inappropriate Use[Title/Abstract]) AND (((((Aged [MeSH Terms OR (Aged, 80 and over[MeSH Terms])) OR elderly[Title/Abstract]) OR older adult[Title/Abstract]) OR older people[Title/Abstract]) Web of Science literature search: TS = ("Drug interactions") TS = ("Inappropriate prescribing" OR "Inappropriate Medication" OR "Inappropriate Medications" OR "Inappropriate Prescription" OR "Inappropriate Prescriptions" OR "Inappropriate Prescribing" OR "Inappropriate Medicines" OR “Inappropriate Drugs" OR "Inappropriate Drug" OR "high-risk medications" OR "Inappropriate Use") TS = (Aged OR Aged, 80 and over OR elderly OR older adult OR older people); Supplementary Material 4. Main characteristics of the studies from general population in primary care, nursing home and hospital settings (IQR* ${ }^{*}$ inter-quartile range).

Author Contributions: Design of the study: M.B. and P.L.C. Screening of the literature search, selection of included studies, and extraction of data: M.B. and P.L.C. Performance of all statistical analyses: G.B. and M.B. Analysis and interpretation of the data: M.B., G.B., M.C. and P.L.C. Drafting of the report: M.B. and P.L.C. All authors have read and agreed to the published version of the manuscript.

Funding: This research received no external funding.

Institutional Review Board Statement: According to the French Data Protection Act of 6 January 1978 , this study was carried-out by the hospital care team and did not require approval from an ethics committee. Authorization to conduct research from the Clinical Data Warehouse of Hospital of Rennes was given by CNIL - Commission Nationale Informatique et Liberté (Authorization number $2020-028$ issued on february 27,2020 ). This study only uses data from existing medical records and did not involve interaction with patients or collection of identifiable private information. Each data sample was deidentified to ensure confidentiality.

Informed Consent Statement: According to the French regulation, hospitalized patients are informed of the use of their hospital health data, and no specific signed consent was required.

Data Availability Statement: Not applicable.

Conflicts of Interest: The authors declare no conflict of interest. 


\section{References}

1. Hines, L.E.; Murphy, J.E. Potentially Harmful Drug-Drug Interactions in the Elderly: A Review. Am. J. Geriatr. Pharmacother. 2011, 9, 364-377. [CrossRef]

2. Magro, L.; Moretti, U.; Leone, R. Epidemiology and characteristics of adverse drug reactions caused by drug-drug interactions. Expert Opin. Drug Saf. 2011, 11, 83-94. [CrossRef] [PubMed]

3. Zanger, U.M.; Schwab, M. Cytochrome P450 enzymes in drug metabolism: Regulation of gene expression, enzyme activities, and impact of genetic variation. Pharmacol. Ther. 2013, 138, 103-141. [CrossRef]

4. Lund, M.; Petersen, T.S.; Dalhoff, K.P. Clinical Implications of P-Glycoprotein Modulation in Drug-Drug Interactions. Drugs 2017, 77, 859-883. [CrossRef]

5. FDA in Vitro Drug Interaction Studies, Cytochrome P450 Enzyme and Transporter Mediated Drug Interactions—Guidance for industry; U.S. Department of Health and Human Services, Center for Drug Evaluation and Research: Silver Spring, MD, USA, 2020; Volume 46.

6. Mallet, L.; Spinewine, A.; Huang, A. The challenge of managing drug interactions in elderly people. Lancet 2007, 370, 185-191. [CrossRef]

7. Hoel, R.W.; Connolly, R.M.G.; Takahashi, P.Y. Polypharmacy Management in Older Patients. Mayo Clin. Proc. 2021, 96, 242-256. [CrossRef]

8. Dechanont, S.; Maphanta, S.; Butthum, B.; Kongkaew, C. Hospital admissions/visits associated with drug-drug interactions: A systematic review and meta-analysis. Pharmacoepidemiol. Drug Saf. 2014, 23, 489-497. [CrossRef]

9. Bénard-Laribière, A.; Miremont-Salamé, G.; Pérault-Pochat, M.-C.; Noize, P.; Haramburu, F.; The EMIR Study Group on behalf of the French network of pharmacovigilance centres. Incidence of hospital admissions due to adverse drug reactions in France: The EMIR study. Fundam. Clin. Pharmacol. 2014, 29, 106-111. [CrossRef]

10. 2019 American Geriatrics Society Beers Criteria ${ }^{\circledR U}$ pdate Expert Panel. American Geriatrics Society 2019 Updated AGS Beers Criteria ${ }^{\circledR} f o r$ Potentially Inappropriate Medication Use in Older Adults. J. Am. Geriatr. Soc. 2019, 67, 674-694. [CrossRef]

11. Moher, D.; Liberati, A.; Tetzlaff, J.; Altman, D.G.; The PRISMA Group. Preferred reporting items for systematic reviews and meta-analyses: The PRISMA statement. PLoS Med. 2009, 6, e1000097. [CrossRef]

12. WHO. World Report on Ageing and Health 2015. Available online: http://www.who.int/ageing/events/world-report-2015 -launch/en/ (accessed on 8 February 2021).

13. Lumley, T. Analysis of Complex Survey Samples. J. Stat. Softw. 2004, 9, 1-19. [CrossRef]

14. Madec, J.; Bouzillé, G.; Riou, C.; Van Hille, P.; Merour, C.; Artigny, M.L.; Delamarre, D.; Raimbert, V.; Lemordant, P.; Cuggia, M. eHOP Clinical Data Warehouse: From a Prototype to the Creation of an Inter-Regional Clinical Data Centers Network. Stud. Health Technol. Inform. 2019, 264, 1536-1537.

15. Bitter, K.; Pehe, C.; Krüger, M.; Heuer, G.; Quinke, R.; Jaehde, U. Pharmacist-led medication reviews for geriatric residents in German long-term care facilities. BMC Geriatr. 2019, 19, 1-8. [CrossRef] [PubMed]

16. Kolar, J.; Tinkova, B.; Ambrus, T.; Tinkova, V. Analysis of Pharmacotherapy in Senior Homes Residents. Acta Pol. Pharm. 2018, $75,223-228$.

17. Fog, A.F.; Kvalvaag, G.; Engedal, K.; Straand, J. Drug-related problems and changes in drug utilization after medication reviews in nursing homes in Oslo, Norway. Scand. J. Prim. Health Care 2017, 35, 329-335. [CrossRef]

18. Alves-Conceição, V.; da Silva, D.T.; de Santana, V.L.; Dos Santos, E.G.; Santos, L.M.; de Lyra, D.P. Evaluation of pharmacotherapy complexity in residents of long-term care facilities: A cross-sectional descriptive study. BMC Pharmacol. Toxicol. 2017, 18, 59. [CrossRef] [PubMed]

19. Lao, C.K.; Ho, S.C.; Chan, K.K.; Tou, C.F.; Tong, H.H.Y.; Chan, A. Potentially inappropriate prescribing and drug-drug interactions among elderly Chinese nursing home residents in Macao. Int. J. Clin. Pharm. 2013, 35, 805-812. [CrossRef]

20. Xavier Pinto, M.C.; Malaquias, D.P.; Ferre, F.; Pimenta Pinheiro, M.L. Potentially inappropriate medication use among institutionalized elderly individuals in southeastern Brazil. Braz. J. Pharm. Sci. 2013, 49, 709-717. [CrossRef]

21. Bakken, M.S.; Ranhoff, A.H.; Engeland, A.; Ruths, S. Inappropriate prescribing for older people admitted to an intermediate-care nursing home unit and hospital wards. Scand. J. Prim. Health Care 2012, 30, 169-175. [CrossRef]

22. Varallo, F.R.; Ambiel, I.S.S.; Nanci, L.O.; Galduróz, J.C.F.; Mastroianni, P.D.C. Assessment of pharmacotherapeutic safety of medical prescriptions for elderly residents in a long-term care facility. Braz. J. Pharm. Sci. 2012, 48, 477-485. [CrossRef]

23. Haasum, Y.; Fastbom, J.; Johnell, K. Institutionalization as a risk factor for inappropriate drug use in the elderly: A Swedish na-tionwide register-based study. Ann. Pharmacother. 2012, 46, 339-346. [CrossRef]

24. Halvorsen, K.H.; Granas, A.G.; Engeland, A.; Ruths, S. Prescribing quality for older people in Norwegian nursing homes and home nursing services using multidose dispensed drugs. Pharmacoepidemiol. Drug Saf. 2011, 21, 929-936. [CrossRef]

25. Hosia-Randell, H.M.V.; Muurinen, S.M.; Pitkälä, K.H. Exposure to potentially inappropriate drugs and drug-drug interactions in elderly nursing home residents in Helsinki, Finland: A cross-sectional study. Drugs Aging 2008, 25, 683-692. [CrossRef]

26. Nygaard, H.A.; Naik, M.; Ruths, S. Nursing-home residents and their drug use: A comparison between mentally intact and mentally impaired residents. Eur. J. Clin. Pharmacol. 2003, 59, 463-469. [CrossRef]

27. Giron, M.S.; Wang, H.X.; Bernsten, C.; Thorslund, M.; Winblad, B.; Fastbom, J. The appropriateness of drug use in an older nonde-mented and demented population. J. Am. Geriatr. Soc. 2001, 49, 277-283. [CrossRef] [PubMed] 
28. Stuhec, M.; Bratović, N.; Mrhar, A. Impact of clinical pharmacist's interventions on pharmacotherapy management in elderly patients on polypharmacy with mental health problems including quality of life: A prospective non-randomized study. Sci. Rep. 2019, 9, 16856-16858. [CrossRef]

29. Bazargan, M.; Smith, J.L.; King, E.O. Potentially inappropriate medication use among hypertensive older African-American adults. BMC Geriatr. 2018, 18, 238. [CrossRef]

30. Allegri, N.; Rossi, F.; Del Signore, F.; Bertazzoni, P.; Bellazzi, R.; Sandrini, G.; Vecchi, T.; Liccione, D.; Pascale, A.; Govoni, S. Drug prescription appropriateness in the elderly: An Italian study. Clin. Interv. Aging 2017, 12, 325-333. [CrossRef]

31. Pasina, L.; Marengoni, A.; Ghibelli, S.; Suardi, F.; Djade, C.D.; Nobili, A.; Franchi, C.; Guerrini, G. A Multicomponent Intervention to Optimize Psycho-tropic Drug Prescription in Elderly Nursing Home Residents: An Italian Multicenter, Prospective, Pilot Study. Drugs Aging 2016, 33, 143-149. [CrossRef]

32. Hanlon, J.T.; Aspinall, S.L.; Handler, S.M.; Gellad, W.F.; Stone, R.A.; Semla, T.P.; Pugh, M.J.V.; Dysken, M.W. Potentially Suboptimal Prescribing for Older Veteran Nursing Home Patients With Dementia. Ann. Pharmacother. 2015, 49, 20-28. [CrossRef]

33. Hanlon, J.T.; Ms, X.W.; Castle, N.G.; Stone, R.A.; Handler, S.M.; Semla, T.P.; Pugh, M.J.; Berlowitz, D.R.; Dysken, M.W. Potential Underuse, Overuse, and Inappropriate Use of Antidepressants in Older Veteran Nursing Home Residents. J. Am. Geriatr. Soc. 2011, 59, 1412-1420. [CrossRef]

34. Bobrova, V.; Heinämäki, J.; Honkanen, O.; Desselle, S.; Airaksinen, M.; Volmer, D. Older adults using multi-dose dispensing exposed to risks of potentially inappropriate medications. Res. Soc. Adm. Pharm. 2019, 15, 1102-1106. [CrossRef] [PubMed]

35. Vatcharavongvan, P.; Puttawanchai, V. Potentially inappropriate medications among the elderly in primary care in Thailand from three different sets of criteria. Pharm. Pr. 2019, 17, 1494. [CrossRef]

36. Toivo, T.; Airaksinen, M.; Dimitrow, M.; Savela, E.; Pelkonen, K.; Kiuru, V.; Suominen, T.; Uunimäki, M.; Kivelä, S.-L.; Leikola, S.; et al. Enhanced coordination of care to reduce medication risks in older home care clients in primary care: A randomized controlled trial. BMC Geriatr. 2019, 19, 1-13. [CrossRef] [PubMed]

37. Stuhec, M.; Gorenc, K.; Zelko, E. Evaluation of a collaborative care approach between general practitioners and clinical pharmacists in primary care community settings in elderly patients on polypharmacy in Slovenia: A cohort retrospective study re-veals positive evidence for implementation. BMC Health Serv. Res. 2019, 19, 118.

38. Patel, R.; Zhu, L.; Sohal, D.; Lenkova, E.; Koshki, N.; Woelfel, J.; Ranson, C.; Valle-Oseguera, C.S.; Rogan, E.L. Use of 2015 Beers Criteria Medications by Older Medicare Beneficiaries. Consult. Pharm. 2018, 33, 48-54. [CrossRef]

39. Novaes, P.H.; da Cruz, D.T.; Lucchetti, A.L.G.; Leite, I.C.G.; Lucchetti, G. The «iatrogenic triad»: Polypharmacy, drug-drug interac-tions, and potentially inappropriate medications in older adults. Int. J. Clin. Pharm. 2017, 39, 818-825. [CrossRef]

40. Hanlon, J.T.; Perera, S.; Newman, A.B.; Thorpe, J.M.; Donohue, J.M.; Simonsick, E.M.; Shorr, R.I.; Bauer, D.C.; Marcum, Z.A.; Health ABC Study. Potential drug-drug and drug-disease in-teractions in well-functioning community-dwelling older adults. J. Clin. Pharm. Ther. 2017, 42, 228-233. [CrossRef]

41. Marin-Gorricho, R.; Casajus-Navasal, A.; Carrillo-Acevedo, L.; Serrano-Perez, A.; Barajas-Santos, M.T.; Hurtado-Gomez, M.F. Analysis of potentially inappropriate prescription according to STOPP/START criteria in a long term care facility. Pharm. Care Esp. 2017, 19, 499-520.

42. Bazargan, M.; Yazdanshenas, H.; Han, S.; Orum, G. Inappropriate Medication Use Among Underserved Elderly African Ameri-cans. J. Aging Health 2016, 28, 118-138. [CrossRef]

43. Popović, B.; Quadranti, N.R.; Matanović, S.M.; Lisica, I.D.; Ljubotina, A.; Duliba, D.P.; Vlahović-Palčevski, V. Potentially inappropriate prescribing in elderly outpatients in Croatia. Eur. J. Clin. Pharmacol. 2014, 70, 737-744. [CrossRef]

44. Tora, H.; Bo, H.; Bodil, L.; Göran, P.; Birgit, E. Potential drug related problems detected by electronic expert support system in patients with multi-dose drug dispensing. Int. J. Clin. Pharm. 2014, 36, 943-952. [CrossRef]

45. Steinman, M.A.; Miao, Y.; Boscardin, W.J.; Komaiko, K.D.R.; Schwartz, J.B. Prescribing Quality in Older Veterans: A Multifocal Approach. J. Gen. Intern. Med. 2014, 29, 1379-1386. [CrossRef]

46. Lund, B.C.; Charlton, M.E.; Steinman, M.A.; Kaboli, P.J. Regional Differences in Prescribing Quality Among Elder Veterans and the Impact of Rural Residence. J. Rural Health 2013, 29, 172-179. [CrossRef]

47. Koper, D.; Kamenski, G.; Flamm, M.; Böhmdorfer, B.; Sönnichsen, A. Frequency of medication errors in primary care patients with polypharmacy. Fam. Pr. 2012, 30, 313-319. [CrossRef]

48. Ghadimi, H.; Esmaily, H.M.; Wahlström, R. General practitioners' prescribing patterns for the elderly in a province of Iran. Pharmacoepidemiol. Drug Saf. 2011, 20, 482-487. [CrossRef]

49. Pozzi, C.; Lapi, F.; Mazzaglia, G.; Inzitari, M.; Boncinelli, M.; Geppetti, P.; Mugelli, A.; Marchionni, N.; Di Bari, M. Is suboptimal prescribing a risk factor for poor health outcomes in community-dwelling elders? The ICARe Dicomano study. Pharmacoepidemiol. Drug Saf. 2010, 19, 954-960. [CrossRef]

50. Johnell, K.; Weitoft, G.R.; Fastbom, J. Sex Differences in Inappropriate Drug Use: A Register-Based Study of Over 600,000 Older People. Ann. Pharmacother. 2009, 43, 1233-1238. [CrossRef]

51. Chrischilles, E.A.; Vangilder, R.; Wright, K.; Kelly, M.; Wallace, R.B. Inappropriate medication use as a risk factor for self-reported adverse drug effects in older adults. J. Am. Geriatr. Soc. 2009, 57, 1000-1006. [CrossRef]

52. Haider, S.I.; Johnell, K.; Weitoft, G.R.; Thorslund, M.; Fastbom, J. The influence of educational level on polypharmacy and inappro-priate drug use: A register-based study of more than 600,000 older people. J. Am. Geriatr. Soc. 2009, 57, 62-69. [CrossRef] 
53. Lapi, F.; Pozzi, C.; Mazzaglia, G.; Ungar, A.; Fumagalli, S.; Marchionni, N.; Geppetti, P.; Mugelli, A.; Di Bari, M. Epidemiology of Suboptimal Prescribing in Older, Community Dwellers. Drugs Aging 2009, 26, 1029-1038. [CrossRef]

54. Johnell, K.; Fastbom, J. Multi-dose drug dispensing and inappropriate drug use: A nationwide register-based study of over 700,000 elderly. Scand. J. Prim. Health Care 2008, 26, 86-91. [CrossRef]

55. Johnell, K.; Fastbom, J.; Rosén, M.; Leimanis, A. Inappropriate Drug Use in the Elderly: A Nationwide Register-Based Study. Ann. Pharmacother. 2007, 41, 1243-1248. [CrossRef]

56. Bregnhøj, L.; Thirstrup, S.; Kristensen, M.B.; Bjerrum, L.; Sonne, J. Prevalence of inappropriate prescribing in primary care. Pharm. World Sci. 2007, 29, 109-115. [CrossRef]

57. Cannon, K.T.; Choi, M.M.; Zuniga, M.A. Potentially inappropriate medication use in elderly patients receiving home health care: A retrospective data analysis. Am. J. Geriatr. Pharmacother. 2006, 4, 134-143. [CrossRef]

58. Zhan, C.; Correa-De-Araujo, R.; Bierman, A.S.; Sangl, J.; Miller, M.R.; Wickizer, S.W.; Stryer, D. Suboptimal Prescribing in Elderly Outpatients: Potentially Harmful Drug-Drug and Drug-Disease Combinations. J. Am. Geriatr. Soc. 2005, 53, 262-267. [CrossRef]

59. Azoulay, L.; Zargarzadeh, A.; Salahshouri, Z.; Oraichi, D.; Bérard, A. Inappropriate medication prescribing in communitydwelling elderly people living in Iran. Eur. J. Clin. Pharmacol. 2005, 61, 913-919. [CrossRef]

60. Tamblyn, R.; Huang, A.; Perreault, R.; Jacques, A.; Roy, D.; Hanley, J.; McLeod, P.; Laprise, R. The medical office of the 21st century (MOXXI): Effec-tiveness of computerized decision-making support in reducing inappropriate prescribing in primary care. CMAJ 2003, 169, 549-556. [PubMed]

61. Schmader, K.; Hanlon, J.T.; Weinberger, M.; Landsman, P.B.; Samsa, G.P.; Lewis, I.; Uttech, K.; Cohen, H.J.; Feussner, J.R. Appropriateness of Medication Prescribing in Ambulatory Elderly Patients. J. Am. Geriatr. Soc. 1994, 42, 1241-1247. [CrossRef]

62. Courlet, P.; Livio, F.; Guidi, M.; Cavassini, M.; Battegay, M.; Stoeckle, M.; Buclin, T.; Alves Saldanha, S.; Csajka, C.; Marzolini, C.; et al. Polypharmacy, Drug-Drug Interactions, and Inap-propriate Drugs: New Challenges in the Aging Population With HIV. Open Forum Infect. Dis. 2019, 6, ofz531. [CrossRef]

63. Lund, J.L.; Sanoff, H.K.; Hinton, S.P.; Muss, H.B.; Pate, V.; Stürmer, T. Potential Medication-Related Problems in Older Breast, Colon, and Lung Cancer Patients in the United States. Cancer Epidemiol. Biomark. Prev. 2017, 27, 41-49. [CrossRef]

64. Oesterhus, R.; Aarsland, D.; Soennesyn, H.; Rongve, A.; Selbaek, G.; Kjosavik, S.R. Potentially inappropriate medications and drug-drug interactions in home-dwelling people with mild dementia. Int. J. Geriatr. Psychiatry 2017, 32, 183-192. [CrossRef]

65. Wucherer, D.; Thyrian, J.R.; Eichler, T.; Hertel, J.; Kilimann, I.; Richter, S.; Michalowsky, B.; Zwingmann, I.; Dreier-Wolfgramm, A.; Ritter, C.A.; et al. Drug-related problems in community-dwelling primary care patients screened positive for dementia. Int. Psychogeriatr. 2017, 29, 1857-1868. [CrossRef]

66. Yazdanshenas, H.; Bazargan, M.; Smith, J.; Martins, D.; Motahari, H.; Orum, G. Pain Treatment of Underserved Older African Americans. J. Am. Geriatr. Soc. 2016, 64, 2116-2121. [CrossRef] [PubMed]

67. Suehs, B.T.; Davis, C.; Franks, B.; Yuran, T.E.; Ng, D.; Bradt, J.; Knispel, J.; Vassilakis, M.; Berner, T. Effect of Potentially Inappropriate Use of Antimuscarinic Medi-cations on Healthcare Use and Cost in Individuals with Overactive Bladder. J. Am. Geriatr. Soc. 2016, 64, 779-787. [CrossRef]

68. Dionne, P.-A.; Vasiliadis, H.-M.; Latimer, E.; Berbiche, D.; Preville, M. Economic Impact of Inappropriate Benzodiazepine Prescribing and Related Drug Interactions Among Elderly Persons. Psychiatr. Serv. 2013, 64, 331-338. [CrossRef]

69. Gallagher, L.P. The potential for adverse drug reactions in elderly patients. Appl. Nurs. Res. 2001, 14, 220-224. [CrossRef] [PubMed]

70. Al-Azayzih, A.; Alamoori, R.; Altawalbeh, S.M. Potentially inappropriate medications prescribing according to Beers criteria among elderly outpatients in Jordan: A cross sectional study. Pharm. Pr. 2019, 17, 1439. [CrossRef]

71. Ertuna, E.; Arun, M.Z.; Ay, S.; Koçak, F.; Özge, K.; Gökdemir, B.; Ispirli, G. Evaluation of pharmacist interventions and commonly used medications in the geriatric ward of a teaching hospital in Turkey: A retrospective study. Clin. Interv. Aging 2019, 14, 587-600. [CrossRef]

72. Oliveira SBV de Barroso, S.C.C.; Bicalho, M.A.C.; Reis, A.M.M. Profile of drugs used for self-medication by elderly attended at a referral center. Einstein 2018, 16, eAO4372. [CrossRef] [PubMed]

73. Colombo, F.; Nunnari, P.; Ceccarelli, G.; Romano, A.V.; Barbieri, P.; Scaglione, F. Measures of Drug Prescribing at Care Transitions in an Internal Medicine Unit. J. Clin. Pharmacol. 2018, 58, 1171-1183. [CrossRef]

74. Ruiz-Millo, O.; Climente-Martí, M.; Navarro-Sanz, J.R. Improvement on prescribing appropriateness after implementing an interdisciplinary pharmacotherapy quality programme in a long-term care hospital. Eur. J. Hosp. Pharm. 2018, 25, 267-273. [CrossRef]

75. Saqib, A.; Atif, M.; Scahill, S. Drug utilization evaluation among an elderly population: A retrospective cross-sectional study in a tertiary care hospital in Pakistan. J. Pharm. Health Serv. Res. 2018, 9, 123-132. [CrossRef]

76. Gutiérrez-Valencia, M.; Izquierdo, M.; Malafarina, V.; Alonso-Renedo, J.; González-Glaría, B.; Larrayoz-Sola, B.; Monforte-Gasque, M.P.; Latasa-Zamalloa, P.; Martinez-Velilla, N. Impact of hospitalization in an acute geriatric unit on polypharmacy and potentially inappropriate prescriptions: A retrospective study. Geriatr. Gerontol. Int. 2017, 17, 2354-2360. [CrossRef]

77. Franchi, C.; Tettamanti, M.; Djade, C.D.; Pasina, L.; Mannucci, P.M.; Onder, G.; Gussoni, G.; Manfellotto, D.; Bonassi, S.; Salerno, F.; et al. E-learning in order to improve drug prescription for hospitalized older patients: A cluster-randomized controlled study. Br. J. Clin. Pharmacol. 2016, 82, 53-63. [CrossRef] 
78. De Melo, D.O.; Storpirtis, S.; Ribeiro, E. Does hospital admission provide an opportunity for improving pharmacotherapy among elderly inpatients? Braz. J. Pharm. Sci. 2016, 52, 391-401. [CrossRef]

79. Grion, A.M.; Gallo, U.; Tinjala, D.D.; Daragjati, J.; Loreggian, M.; Cardaci, G.; Mangoni, A.; Pilotto, A. A New ComputerBased Tool to Reduce Potentially Inappropriate Prescriptions in Hospitalized Geriatric Patients. Drugs Aging 2016, 33, 267-275. [CrossRef] [PubMed]

80. Salwe, K.J.; Kalyansundaram, D.; Bahurupi, Y. A Study on Polypharmacy and Potential Drug-Drug Interactions among Elderly Patients Admitted in Department of Medicine of a Tertiary Care Hospital in Puducherry. J. Clin. Diagn. Res. 2016, 10, FC06-FC10. [CrossRef]

81. Matanović, S.M.; Vlahović-Palčevski, V. Potentially inappropriate prescribing to the elderly: Comparison of new protocol to Beers criteria with relation to hospitalizations for ADRs. Eur. J. Clin. Pharmacol. 2014, 70, 483-490. [CrossRef]

82. Franchi, C.; On behalf of ELICADHE Investigators; Mari, D.; Tettamanti, M.; Pasina, L.; Djade, C.D.; Mannucci, P.M.; Onder, G.; Bernabei, R.; Gussoni, G.; et al. E-learning to improve the drug prescribing in the hospitalized elderly patients: The ELICADHE feasibility pilot study. Aging Clin. Exp. Res. 2013, 26, 435-443. [CrossRef]

83. Ghibelli, S.; Marengoni, A.; Djade, C.D.; Nobili, A.; Tettamanti, M.; Franchi, C.; Caccia, S.; Giovarruscio, F.; Remuzzi, A.; Pasina, L.; et al. Prevention of inappropriate prescribing in hos-pitalized older patients using a computerized prescription support system (INTERcheck(®)). Drugs Aging 2013, 30, 821-828. [CrossRef]

84. Siqueira, J.S.; Antoniolli, A.R.; Silvestre, C.C.; Silva, D.T.; Serafini, M.R.; Oliveira-Filho, A.D.; Lyra, D.P., Jr. Rational drug prescribing for elderly inpatients in a Brazilian hospital: A pilot study. Afr. J. Pharm. Pharmacol. 2012, 6, 877-882.

85. Trivalle, C.; Cartier, T.; Verny, C.; Mathieu, A.M.; Davrinche, P.; Agostini, H.; Becquemont, L.; Demolis, P. Identifying and preventing adverse drug events in elderly hospitalised patients: A randomised trial of a program to reduce adverse drug effects. J. Nutr. Health Aging 2010, 14, 57-61. [CrossRef]

86. Schuler, J.; Dückelmann, C.; Beindl, W.; Prinz, E.; Michalski, T.; Pichler, M. Polypharmacy and inappropriate prescribing in elderly internal-medicine patients in Austria. Wien. Klin. Wochenschr. 2008, 120, 733-741. [CrossRef]

87. Nixdorff, N.; Hustey, F.M.; Brady, A.K.; Vaji, K.; Leonard, M.; Messinger-Rapport, B.J. Potentially inappropriate medications and adverse drug effects in elders in the ED. Am. J. Emerg. Med. 2008, 26, 697-700. [CrossRef] [PubMed]

88. Radosević, N.; Gantumur, M.; Vlahović-Palcevski, V. Potentially inappropriate prescribing to hospitalised patients. Pharmacoepidemiol. Drug Saf. 2008, 17, 733-737. [CrossRef] [PubMed]

89. Saltvedt, I.; Spigset, O.; Ruths, S.; Fayers, P.; Kaasa, S.; Sletvold, O. Patterns of drug prescription in a geriatric evaluation and man-agement unit as compared with the general medical wards: A randomised study. Eur. J. Clin. Pharmacol. 2005, 61, 921-928. [CrossRef] [PubMed]

90. Fillenbaum, G.G.; Hanlon, J.T.; Landerman, L.R.; Artz, M.B.; O'Connor, H.; Dowd, B.; Gross, C.R.; Boult, C.; Garrard, J.; Schmader, K.E. Impact of inappropriate drug use on health services utilization among representative older community-dwelling residents. Am. J. Geriatr. Pharmacother. 2004, 2, 92-101. [CrossRef]

91. Frank, C.; Godwin, M.; Verma, S.; Kelly, A.; Birenbaum, A.; Seguin, R.; Anderson, J. What drugs are our frail elderly patients taking? Do drugs they take or fail to take put them at increased risk of interactions and inappropriate medication use? Can. Fam. Physician 2001, 47, 1198-1204.

92. Jørgensen, T.L.; Herrstedt, J. The influence of polypharmacy, potentially inappropriate medications, and drug interactions on treatment completion and prognosis in older patients with ovarian cancer. J. Geriatr. Oncol. 2020, 11, 593-602. [CrossRef]

93. Hong, S.; Lee, J.H.; Chun, E.K.; Kim, K.I.; Kim, J.W.; Kim, S.H.; Lee, Y.G.; Hwang, I.G.; Kim, J.Y.; Koh, S.J.; et al. Polypharmacy, Inappropriate Medication Use, and Drug Interac-tions in Older Korean Patients with Cancer Receiving First-Line Palliative Chemotherapy. Oncologist 2020, 25, e502. [CrossRef]

94. LaVan, A.; O'Mahony, D.; Buckley, M.; O'Mahony, D.; Gallagher, P. 150 Severe Drug Interactions (SDIs) and Potentially Inappropriate Prescriptions (PIPs) in Older Adults with Cancer. Age Ageing 2019, 48, iii1-iii16. [CrossRef]

95. Graf, J.; Lucke, T.; Herrera, R.; Watz, H.; Holle, R.; Vogelmeier, C.; Ficker, J.H.; Jörres, R.A. Compatibility of medication with PRISCUS criteria and identification of drug interactions in a large cohort of patients with COPD. Pulm. Pharmacol. Ther. 2018, 49, 123-129. [CrossRef]

96. Leger, D.Y.; Moreau, S.; Signol, N.; Fargeas, J.-B.; Picat, M.-A.; Penot, A.; Abraham, J.; Laroche, M.-L.; Bordessoule, D. Polypharmacy, potentially inappropriate medications and drug-drug interactions in geriatric patients with hematologic malignancy: Observational single-center study of 122 patients. J. Geriatr. Oncol. 2018, 9, 60-67. [CrossRef]

97. Alkan, A.; Yaşar, A.; Karc1, E.; Köksoy, E.B.; Ürün, M.; Şenler, F. Çay; Ürün, Y.; Tuncay, G.; Ergün, H.; Akbulut, H. Severe drug interactions and potentially inappropriate medication usage in elderly cancer patients. Support. Care Cancer 2016, 25, 229-236. [CrossRef]

98. Bugat, M.E.; Bourgouin, M.; Gérard, S.; Lozano, S.; Brechemier, D.; Cestac, P.; Cool, C.; Balardy, L. Drug Prescription Including Interactions with Anticancer Treatments in the Elderly: A Global Approach. J. Nutr. Health Aging 2017, 21, 849-854. [CrossRef]

99. Vrijkorte, E.; De Vries, J.; Schaafsma, R.; Wymenga, M.; Munnink, T.O. Optimising pharmacotherapy in older cancer patients with polypharmacy. Eur. J. Cancer Care 2019, 29, e13185. [CrossRef]

100. Parian, A.; Ha, C.Y. Older Age and Steroid Use Are Associated with Increasing Polypharmacy and Potential Medication Interactions Among Patients with Inflammatory Bowel Disease. Inflamm. Bowel Dis. 2015, 21, 1392-1400. [CrossRef] 
101. Jarchow-MacDonald, A.A.; Mangoni, A.A. Prescribing patterns of proton pump inhibitors in older hospitalized patients in a Scottish health board. Geriatr. Gerontol. Int. 2013, 13, 1002-1009. [CrossRef]

102. Hoffmann, W.; Berg, N.; Thyrian, J.R.; Fiss, T. Frequency and determinants of potential drug-drug interactions in an elderly population receiving regular home visits by GPs-Results of the home medication review in the AGnES-studies. Pharmacoepidemiol. Drug Saf. 2011, 20, 1311-1318. [CrossRef]

103. Rodríguez-Terol, A.; Caraballo, M.; Palma, D.; Santos-Ramos, B.; Molina, T.; Desongles, T.; Aguilar, A. Quality of interaction database management systems. Farm. Hosp. Organo Of. Expr. Cient. Soc. Esp. Farm. Hosp. 2009, 33, 134-146. [CrossRef]

104. CheckTheMeds. Available online: https://www.checkthemeds.com/ (accessed on 5 October 2020).

105. Drug Interactions Checker-For Drugs, Food \& Alcohol. Available online: https://www.drugs.com/drug_interactions.html (accessed on 14 December 2020).

106. Drug Interaction Database. FDB (First Databank). Available online: https://www.fdbhealth.com/solutions/medknowledgedrug-database/medknowledge-clinical-modules/drug-drug-interaction (accessed on 6 October 2020).

107. Drug Interactions by Hansten and Horn. Available online: http:/ / www.hanstenandhorn.com/book_top100.htm (accessed on 5 October 2020).

108. Hansten, P.D.; Horn, J.R.; Hazlet, T.K. ORCA: OpeRational ClassificAtion of drug interactions. J. Am. Pharm. Assoc. 2001, 41, 161-165. [CrossRef]

109. What to Know About Drug Interactions. Healthline. 2020. Available online: https://www.healthline.com/health/druginteractions (accessed on 5 October 2020).

110. Guida all'uso dei Farmaci. Available online: https:/ / www.aifa.gov.it/sites/default/files/bif0305-6252.pdf (accessed on 6 October 2020).

111. Lexicomp Clinical Drug Information. Available online: https:/ /www.wolterskluwercdi.com/lexicomp-online/ (accessed on 5 October 2020).

112. Drug Interactions Checker-Medscape Drug Reference Database. Available online: https://reference.medscape.com/druginteractionchecker (accessed on 5 October 2020).

113. Home-MICROMEDEX. 2018. Available online: http:/ /www.micromedexsolutions.com/micromedex2/librarian/ (accessed on 2 August 2018).

114. About MIMS NZ Interactions. Available online: http:/ / www.mims.co.nz/AboutMIMSInteractions.aspx (accessed on 5 October 2020).

115. The Norwegian Pharmaceutical Product Compendium. Available online: https://www.felleskatalogen.no/m/medisin/ dokument/markedsforing-brosjyre.pdf (accessed on 5 October 2020).

116. Intelligent Pharmacy Software vs. PharmASSIST. Available online: https://www.capterra.com/pharmacy-software/compare/15 5478-106398/Intelligent-Pharmacy-Software-vs-PharmASSIST (accessed on 5 October 2020).

117. Şimşek, A.; Taner, N.; Macit, Ç.; Berk, B.; Mercanoğlu, G. The Importance of Computerized Drug Interaction Checker Programs Used in Community Pharmacies to Avoid Potential Drug Interactions: A Preliminary Study with Clarithromycin. Potansiyel ilaç Etkileşimlerinin Önlenmesinde Serbest Eczanelerde Kullanılan Bilgisayarlı Ilaç Etkileşimi Kontrol Programlarının Önemi: Klaritromisin ile Yapılan bir ön Çalışma. 2019. Available online: http:/ /acikerisim.medipol.edu.tr/xmlui/handle/20.500.12511/4681 (accessed on 5 October 2020).

118. Sönnerstam, E.; Sjölander, M.; Lövheim, H.; Gustafsson, M. Clinically relevant drug-drug interactions among elderly people with dementia. Eur. J. Clin. Pharmacol. 2018, 74, 1351-1360. [CrossRef]

119. Baysari, M.T.; Zheng, W.Y.; Li, L.; Westbrook, J.; Day, R.O.; Hilmer, S.; Van Dort, B.A.; Hargreaves, A.; Kennedy, P.; Monaghan, C.; et al. Optimising computerised decision support to transform medication safety and reduce prescriber burden: Study protocol for a mixed-methods evaluation of drug-drug interaction alerts. BMJ Open 2019, 9, e026034. [CrossRef]

120. Husson, M.-C. Theriaque: Independent-drug database for good use of drugs by health practitioners. Ann. Pharm. Fr. 2008, 66, 268-277. [CrossRef]

121. Liverpool HIV Interactions. Available online: https://www.hiv-druginteractions.org/checker (accessed on 5 October 2020).

122. Chang, S.H.; Chou, I.J.; Yeh, Y.H.; Chiou, M.J.; Wen, M.S.; Kuo, C.T.; See, L.C.; Kuo, C.F. Association Between Use of Non-Vitamin K Oral Anti-coagulants With and Without Concurrent Medications and Risk of Major Bleeding in Nonvalvular Atrial Fibrillation. JAMA 2017, 318, 1250-1259. [CrossRef]

123. Niemi, M.; Backman, J.T.; Neuvonen, M.; Laitila, J.; Neuvonen, P.J.; Kivistö, K.T. Effects of fluconazole and fluvoxamine on the pharmacokinetics and pharmacodynamics of glimepiride. Clin. Pharmacol. Ther. 2001, 69, 194-200. [CrossRef]

124. Pérez, T.; Moriarty, F.; Wallace, E.; McDowell, R.; Redmond, P.; Fahey, T. Prevalence of potentially inappropriate prescribing in older people in primary care and its association with hospital admission: Longitudinal study. BMJ 2018, 363, k4524. [CrossRef]

125. Chang, C.-B.; Chen, J.-H.; Wen, C.-J.; Kuo, H.-K.; Lu, I.-S.; Chiu, L.-S.; Wu, S.-C.; Chan, D.-C. (Derrick) Potentially inappropriate medications in geriatric outpatients with polypharmacy: Application of six sets of published explicit criteria. Br. J. Clin. Pharmacol. 2011, 72, 482-489. [CrossRef]

126. Hukins, D.; MacLeod, U.; Boland, J.W. Identifying potentially inappropriate prescribing in older people with dementia: A systematic review. Eur. J. Clin. Pharmacol. 2019, 75, 467-481. [CrossRef]

127. Fung, K.W.; Kapusnik-Uner, J.; Cunningham, J.; Higby-Baker, S.; Bodenreider, O. Comparison of three commercial knowledge bases for detection of drug-drug interactions in clinical decision support. J. Am. Med. Inform. Assoc. 2017, 24, 806-812. [CrossRef] [PubMed]

128. Morival, C.; Westerlynck, R.; Bouzillé, G.; Cuggia, M.; Le Corre, P. Prevalence and nature of statin drug-drug interactions in a university hospital by electronic health record mining. Eur. J. Clin. Pharmacol. 2018, 74, 525-534. [CrossRef] [PubMed]

129. Zheng, W.Y.; Richardson, L.C.; Li, L.; Day, R.O.; Westbrook, J.I.; Baysari, M.T. Drug-drug interactions and their harmful effects in hospitalised patients: A systematic review and meta-analysis. Eur. J. Clin. Pharmacol. 2018, 74, 15-27. [CrossRef] 
130. Le Corre, P.A. Prescriptome analytics: An opportunity for clinical pharmacy. Int. J. Clin. Pharm. 2019, 41, 1394-1397. [CrossRef]

131. McDonnell, P.J.; Jacobs, M.R. Hospital admissions resulting from preventable adverse drug reactions. Ann. Pharmacother. 2002, 36, 1331-1336. [CrossRef] [PubMed] 